Type: Letter/Methods

2

selscan 2 . 0: scanning for sweeps in unphased data

Zachary A. Szpiech ${ }^{1,2,}$

${ }^{1}$ Department of Biology, Pennsylvania State University, University Park, PA 16802, USA

$8{ }^{2}$ Institute for Computational and Data Sciences, Pennsylvania State University, University Park, 9 PA 16802, USA

10 "Correspondence: szpiech@psu.edu

Abstract

Haplotype-based scans to identify recent and ongoing positive selection have become commonplace in evolutionary genomics studies of numerous species across the tree of life.

15 However, the most widely adopted approaches require phased haplotypes to compute the key

16 statistics. Here we release a major update to the selscan software that re-defines popular

17 haplotype-based statistics for use with unphased "multi-locus genotype" data. We provide

18 unphased implementations of iHS, nSL, XP-EHH, and XP-nSL and evaluate their performance

19 across a range of important parameters in a generic demographic history. Source code and

20 executables are available at https://www.github.com/szpiech/selscan.

\title{
211 Introduction
}

Haplotype-based summary statistics—such as iHS (Voight, et al. 2006), nSL (Ferrer-

23 Admetlla, et al. 2014), XP-EHH (Sabeti, et al. 2007), and XP-nSL (Szpiech, et al. 2021)—have

24 become commonplace in evolutionary genomics studies to identify recent and ongoing positive

25 selection in populations (e.g.,Colonna, et al. 2014; Zoledziewska, et al. 2015; Nedelec, et al.

26 2016; Crawford, et al. 2017; Meier, et al. 2018; Lu, et al. 2019; Zhang, et al. 2020; Salmon, et al.

27 2021). When an adaptive allele sweeps through a population, it leaves a characteristic pattern

28 of long high-frequency haplotypes and low genetic diversity in the vicinity of the allele. These

29 statistics aim to capture these signals by summarizing the decay of haplotype homozygosity as

30 a function of distance from a putatively selected region, either within a single population (iHS 
31 and $n S L)$ or between two populations (XP-EHH and XP-nSL). However, each of these statistics

32 presumes that haplotype phase is known.

33 Recent work has shown that converting haplotype data into multi-locus genotype data is

34 an effective approach for using haplotype-based selection statistics such as G12, LASSI, and

35 saltiLASSI (Harris, et al. 2018; Harris and DeGiorgio 2020; DeGiorgio and Szpiech 2021) in

36 unphased data. Recognizing this, we have reformulated the iHS, nSL, XP-EHH, and XP-nSL

37 statistics to use multi-locus genotypes and provided an easy-to-use implementation in selscan

382.0 (Szpiech and Hernandez 2014). We also evaluate the performance of these unphased

39 statistics under various generic demographic models.

\section{New Approaches}

41 When the --unphased flag is set in selscan v2.0+, biallelic genotype data is collapsed

42 into multi-locus genotype data by representing the genotype as either 0,1 , or 2 -the number of

43 derived alleles observed. In this case, selscan v2.0+ will then compute iHS, nSL, XP-EHH, and

$44 \mathrm{XP}-\mathrm{nSL}$ as described below. We follow the notation conventions of Szpiech and Hernandez

45 (2014).

\subsection{Extended Haplotype Homozygosity}

47 In a sample of $n$ diploid individuals, let $\mathcal{C}$ denote the set of all possible genotypes at

48 locus $x_{0}$. For multi-locus genotypes, $\mathcal{C}:=\{0,1,2\}$, representing the total counts of a derived

49 allele. Let $\mathcal{C}\left(x_{i}\right)$ be the set of all unique haplotypes extending from site $x_{0}$ to site $x_{i}$ either

50 upstream or downstream of $x_{0}$. If $x_{1}$ is a site immediately adjacent to $x_{0}$, then $\mathcal{C}\left(x_{1}\right):=$

$51\{00,01,02,10,11,12,20,21,22\}$, representing all possible two-site multi-locus genotypes. We can

52 then compute the extended haplotype homozygosity $(\mathrm{EHH})$ of a set of multi-locus genotypes as

$$
\operatorname{EHH}\left(x_{i}\right) \sum_{h \in \mathcal{C}\left(x_{i}\right)} \frac{\left(\begin{array}{c}
n_{h} \\
2
\end{array}\right)}{\left(\begin{array}{c}
n \\
2
\end{array}\right)},
$$

54 where $n_{h}$ is the number of observed haplotypes of type $h$. 
If we wish to compute the EHH of a subset of observed haplotypes that all contain the

56 same 'core' multi-locus genotype, let $\mathcal{H}_{c}\left(x_{i}\right)$ be the partition of $\mathcal{C}\left(x_{i}\right)$ containing genotype $c \in \mathcal{C}$

57 at $x_{0}$. For example, choosing a homozygous derived genotype $(c=2)$ as the core, $\mathcal{H}_{2}:=$

$58\{20,21,22\}$. Thus, we can compute the $\mathrm{EHH}$ of all individuals carrying a given genotype at site $x_{0}$

59 extending out to site $x_{i}$ as

60

$$
E H H_{c}\left(x_{i}\right)=\sum_{h \in \mathcal{H}_{c}\left(x_{i}\right)} \frac{\left(\begin{array}{c}
n_{h} \\
2
\end{array}\right)}{\left(\begin{array}{c}
n_{c} \\
2
\end{array}\right)}
$$

61 where $n_{h}$ is the number of observed haplotypes of type $h$ and $n_{c}$ is the number of observed

62 multi-locus genotypes with core genotype of $c$. Finally, we can compute the complement EHH of

63 a sample of multi-locus genotypes as

$$
c E H H_{c}\left(x_{i}\right)=\sum_{h \in \mathcal{C}\left(x_{i}\right) \backslash \mathcal{H}_{c}\left(x_{i}\right)} \frac{\left(\begin{array}{c}
n_{h} \\
2
\end{array}\right)}{\left(\begin{array}{c}
n_{c^{\prime}} \\
2
\end{array}\right)}
$$

65 where $n_{c^{\prime}}$ is the number of observed multi-locus genotypes with a core genotype of not $c$.

\section{$66 \quad 2.2 \mathrm{iHS}$ and $\mathrm{nSL}$}

67 Unphased iHS and nSL are calculated using the equations above. First, we compute the 68 integrated haplotype homozygosity $(\mathrm{iHH})$ for the homozygous ancestral $(c=0)$ and derived $(c=$

69 2) core genotypes as

$$
i H H_{c}=\sum_{i=1}^{|\mathcal{D}|} \frac{1}{2}\left(E H H_{c}\left(x_{i-1}\right)+E H H_{c}\left(x_{i}\right)\right) \mathrm{d}\left(x_{i-1}, x_{i}\right)+\sum_{i=1}^{|u|} \frac{1}{2}\left(E H H_{c}\left(x_{i-1}\right)+E H H_{c}\left(x_{i}\right)\right) \mathrm{d}\left(x_{i-1}, x_{i}\right),
$$

71 where $\mathcal{D}$ is the set of downstream sites from the core locus and $\mathcal{U}$ is the set of upstream sites.

$72 d\left(x_{i-1}, x_{i}\right)$ is a measure of genomic distance between to markers and is the genetic distance in

73 centimorgans or physical distance in basepairs for iHS (Voight, et al. 2006) or the number of

74 sites observed for nSL (Ferrer-Admetlla, et al. 2014). We similarly compute the complement

75 integrated haplotype homozygosity $(\mathrm{ciHH})$ for both homozygous core genotypes as 


$$
+\sum_{i=1}^{|u|} \frac{1}{2}\left(\mathrm{c} E H H_{c}\left(x_{i-1}\right)+\mathrm{c} E H H_{c}\left(x_{i}\right)\right) \mathrm{d}\left(x_{i-1}, x_{i}\right)
$$

78 The (unstandardized) unphased iHS is then calculated as

$$
i H S=\left\{\begin{array}{lr}
\mathrm{iHS}_{2}, & \text { if } \mathrm{iHS}_{2}>\mathrm{iHS}_{0} \\
-\mathrm{iHS}_{0}, & \text { otherwise }
\end{array}\right.
$$

80 where $i H S_{2}=\log _{10}\left(\frac{i H H_{2}}{c i H H_{2}}\right)$ and $i H S_{0}=\log _{10}\left(\frac{i H H_{0}}{c i H H_{0}}\right)$. Unstandardized iHS scores are then

81 normalized in frequency bins, as previously described (Voight, et al. 2006; Ferrer-Admetlla, et

82 al. 2014). Unstandardized unphased nSL is computed similarly with the appropriate distance

83 measure. Large positive scores indicate long high-frequency haplotypes with a homozygous

84 derived core genotype, and large negative scores indicate long high-frequency haplotypes with

85 a homozygous ancestral core genotype. Clusters of extreme scores in both directions indicate

86 evidence for a sweep.

\section{$87 \quad 2.3$ XP-EHH and XP-nSL}

89 populations $A$ and $B$, using the entire sample in each population. $\mathrm{iHH}$ in a population $\mathrm{P}$ is

90 computed as

$$
i H H_{\mathrm{P}}=\sum_{i=1}^{|\mathcal{D}|} \frac{1}{2}\left(\operatorname{EHH}\left(x_{i-1}\right)+\operatorname{EHH}\left(x_{i}\right)\right) \mathrm{d}\left(x_{i-1}, x_{i}\right)+\sum_{i=1}^{|u|} \frac{1}{2}\left(\operatorname{EHH}\left(x_{i-1}\right)+\operatorname{EHH}\left(x_{i}\right)\right) \mathrm{d}\left(x_{i-1}, x_{i}\right),
$$

92 where the distance measure is given as centimorgans or basepairs for XP-EHH (Sabeti, et al.

93 2007) and number of sites observed for XP-nSL (Szpiech, et al. 2021). The XP statistics

94 between population $A$ and $B$ are then computed as $X P=\log _{10}\left(\frac{i H H_{A}}{i H H_{B}}\right)$ and are normalized

95 genome wide in a single bin. Large positive scores indicate long high-frequency haplotypes in 
96 population $A$, and large negative scores indicate long high-frequency haplotypes in population

97 B. Clusters of extreme scores in one direction indicate evidence for a sweep in that population.

\section{Methods}

\section{$99 \quad 3.1$ Simulations}

We evaluate the performance of the unphased versions of iHS, nSL, XP-EHH, and XP-

101 nSL under a generic two-population divergence model using the coalescent simulation program

102 discoal (Kern and Schrider 2016). We explore five versions of this generic model and name

103 them Demo 1 through Demo 5 (Table 1). Let $N_{0}$ and $N_{1}$ be the effective population sizes of

104 Population 0 and Population 1 after the split from their ancestral population (of size $N_{A}$ ). For

105 Demo 1, we keep a constant population size post-split and let $N_{0}=N_{1}=10,000$. For Demo 2,

106 we keep a constant population size post-split and let $N_{0}=2 N_{1}=10,000$. For Demo 3, we keep

107 a constant population size post-split and let $2 N_{0}=N_{1}=10,000$. For Demo 4, we initially set

$108 N_{0}=N_{1}=10,000$ and let $N_{0}$ grow stepwise exponentially every 50 generations starting at 2,000

109 generations ago until $N_{0}=5 N_{1}=50,000$. For Demo 5 , we initially set $N_{0}=N_{1}=10,000$ and let

$110 N_{1}$ grow stepwise exponentially every 50 generations starting at 2,000 generations ago until

$1115 N_{0}=N_{1}=50,000$.

112 For each demographic history we vary the population divergence time $t_{d} \in$

$113\{2000,4000,8000\}$ generations ago. For non-neutral simulations, we simulate a sweep in

114 Population 0 in the middle of the simulated region across a range of selection coefficients $s \in$

$115\{0.005,0.01,0.02\}$. We vary the frequency at which the adaptive allele starts sweeping as $e \in$

$116\{0,0.01,0.02,0.05,0.10\}$, where $e=0$ indicates a hard sweep and $e>0$ indicates a soft sweep,

117 and we also vary the frequency of the selected allele at time of sampling $f \in\{0.7,0.8,0.9,1.0\}$

118 as well as $g \in\{50,100\}$ representing fixation of the sweeping allele $g$ generations ago. For all

119 simulations we set the genome length to be $L=500,000$ basepairs, the ancestral effective

120 population size to be $N_{A}=10,000$, the per site per generation mutation rate at $\mu=2.35 \times 10^{-8}$, 
121 and the per site per generation recombination rate at $r=1.2 \times 10^{-8}$. For neutral simulations, we

122 simulate 1,000 replicates for each parameter set, and for non-neutral simulations we simulate

123100 replicates for each parameter set. As iHS and nSL are single population statistics, we only

124 analyze Demo 1, Demo 3, and Demo 4 with these statistics, as Demo 2 and Demo 5 have a

125 constant size history identical to Demo 1 for Population 0, where the sweeps are simulated.

126 For all simulations, we compute the relevant statistics (--ihs, --nsl, --xpehh, or --xpnsl)

127 with selscan v2.0, using the --unphased and --trunc-ok flags. For iHS and XP-EHH, we also use

128 the --pmap flag in order to use physical distance instead of a recombination map.

\section{$129 \quad 3.2$ Power and False Positive Rate}

To compute power for iHS and nSL, we follow the approach of Voight et al. (2006). For

131 these statistics, each non-neutral replicate is individually normalized jointly with all matching

132 neutral replicates in $1 \%$ allele frequency bins. Because extreme values of the statistic are likely

133 to be clustered along the genome (Voight, et al. 2006), we then compute the proportion of

134 extreme scores $(|i H S|>2$ or $|n S L|>2)$ within 100kbp non-overlapping windows. We then bin

135 these windows into 10 quantile bins based on the number of scores observed in each window

136 and call the top $1 \%$ of these windows as putatively under selection. We calculate the proportion

137 of non-neutral replicates that fall in this top $1 \%$ as the power. To compute the false positive rate,

138 we compute the proportion of neutral simulations that fall within the top $1 \%$.

139 To compute power for XP-EHH and XP-nSL, we follow the approach of Szpiech et al.

140 (2021). For these statistics, each non-neutral replicate is individually normalized jointly with all

141 matching neutral replicates. Because extreme values of the statistic are likely to be clustered

142 along the genome (Szpiech, et al. 2021), we then compute the proportion of extreme scores

143 (XP-EHH > 2 or XP-nSL > 2) within 100kbp non-overlapping windows. We then bin these

144 windows into 10 quantile bins based on the number of scores observed in each window and call

145 the top $1 \%$ of these windows as putatively under selection. We calculate the proportion of non- 
146 neutral replicates that fall in this top $1 \%$ as the power. To compute the false positive rate, we

147 compute the proportion of neutral simulations that fall within the top $1 \%$.

\section{$148 \quad 4$ Results}

We find that the unphased versions of iHS and nSL have good power (Figures 1, S1-S4,

150 S13-16, and S21-24) to detect selection prior to fixation of the allele, with nSL generally

151 outperforming iHS. In smaller populations (Figure 1C and 1D), power does suffer relative to

152 larger populations (Figure 1A, 1B, 1E, 1F). Each of these statistics also have low false positive

153 rates hovering around $1 \%$ (Table S1).

154 Similarly, we find that the unphased versions of XP-EHH and XP-nSL have good power

155 as well (Figures 2, 3, S5-S12, S17-S20, and S25-S32). When the sweep takes place in the

156 smaller of the two populations (Figure 2C and 2D), we see a similar decrease in power. When

157 one population is undergoing exponential growth (Figure 3) performance is generally quite

158 good, likely the result of a larger effective selection coefficient in large populations. These two-

159 population statistics generally outperform their single-population counterparts, especially for

160 sweeps that have reached fixation recently. Each of these statistics also have low false positive

161 rates hovering around $1 \%$ (Table S1).

\section{Discussion}

163 We introduce multi-locus genotype versions of four popular haplotype-based selection

164 statistics_iHS (Voight, et al. 2006), nSL (Ferrer-Admetlla, et al. 2014), XP-EHH (Sabeti, et al.

165 2007), and XP-nSL (Szpiech, et al. 2021) — that can be used when the phase of genotypes is

166 unknown. We implement these updates in the latest v2.0 update of the program selscan

167 (Szpiech and Hernandez 2014), with source code and pre-compiled binaries available at

168 https://www.github.com/szpiech/selscan.

\section{Acknowledgements}


bioRxiv preprint doi: https://doi.org/10.1101/2021.10.22.465497; this version posted October 24, 2021. The copyright holder for this preprint (which was not certified by peer review) is the author/funder, who has granted bioRxiv a license to display the preprint in perpetuity. It is made available under aCC-BY-NC-ND 4.0 International license.

171 Department of Biology. Computations for this research were performed using the Pennsylvania

172 State University's Institute for Computational Data Sciences' Roar supercomputer.

173

A

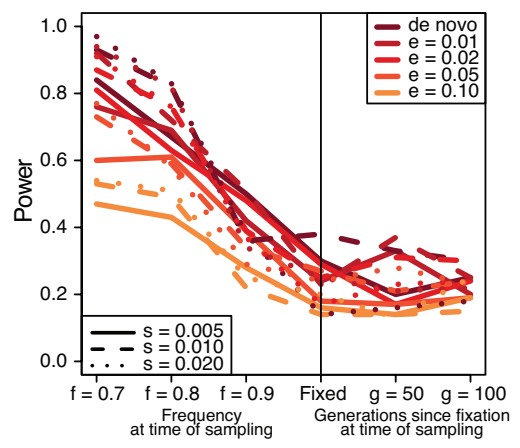

C



E



B $n S L ; t_{d}=4000$

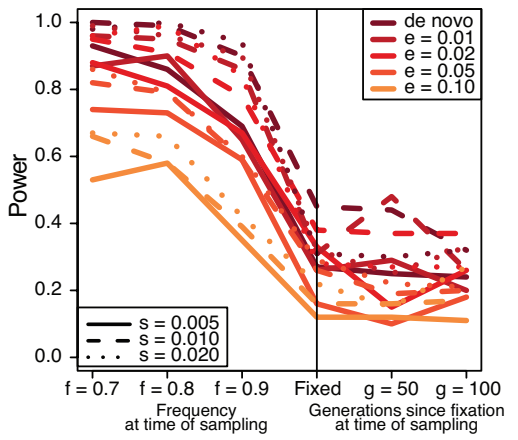

D

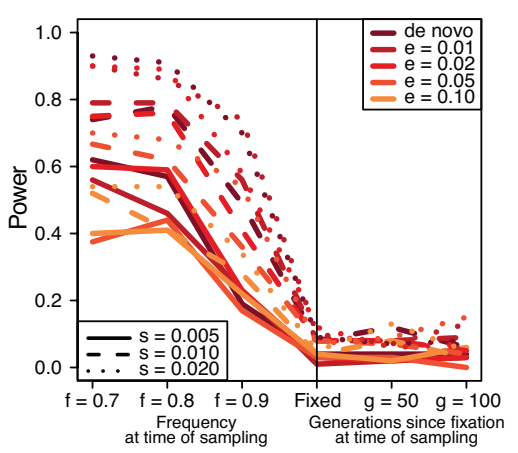

$F$

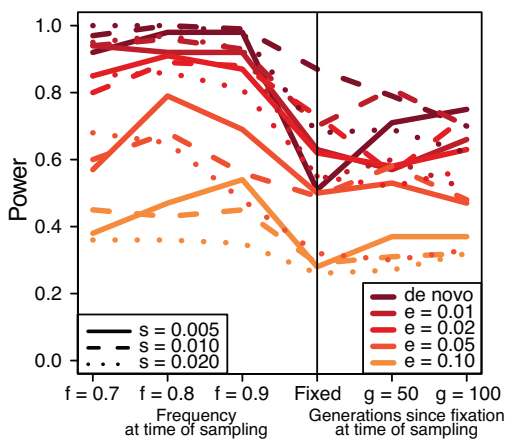

174

Figure 1. Power curves for unphased implementations of iHS $(A, C$, and $E)$ and $n S L(B, D$, and

F) under demographic histories Demo 1 (A and B), Demo 3 (C and D), and Demo 4 (E and F). $s$

177 is the selection coefficient, $f$ is the frequency of the adaptive allele at time of sampling, $g$ is the

178 number of generations at time of sampling since fixation, $e$ is the frequency at which selection

179 began, and $t_{d}$ is the time in generations since the two populations diverged. 




B XP-nSL; $t_{d}=4000$
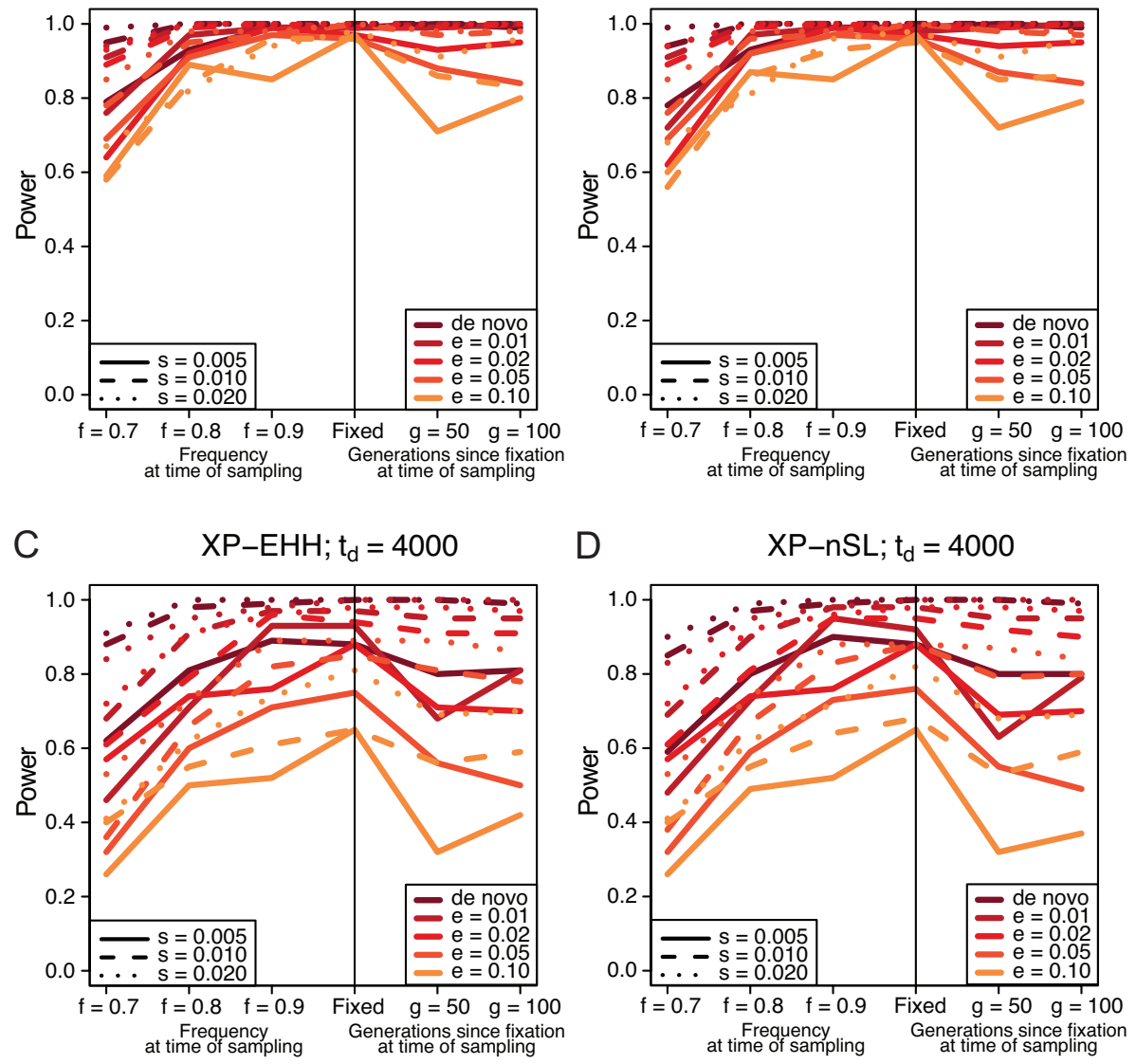

D XP-nSL; $t_{d}=4000$

E

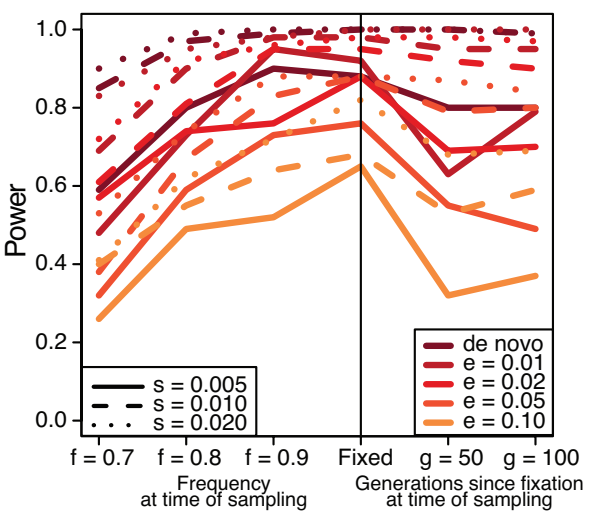

$X P-E H H ; t_{d}=4000$

F
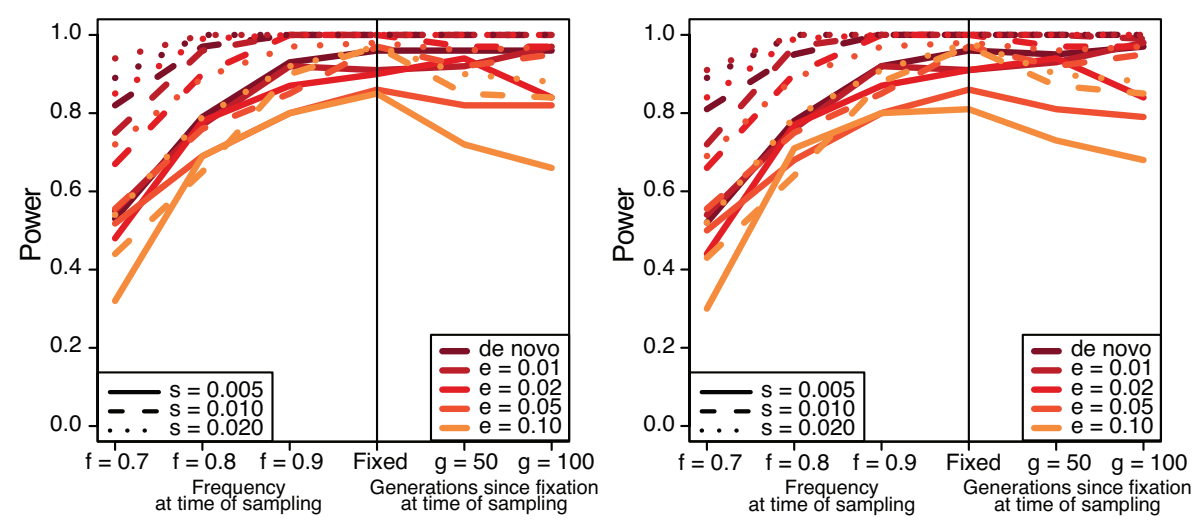

181 Figure 2. Power curves for unphased implementations of XP-EHH (A, C, and E) and XP-nSL

$182(B, D$, and $F)$ under demographic histories Demo 1 ( $A$ and $B)$, Demo 2 ( $C$ and $D)$, and Demo 3

$183(\mathrm{E}$ and $\mathrm{F}) . s$ is the selection coefficient, $f$ is the frequency of the adaptive allele at time of

184 sampling, $g$ is the number of generations at time of sampling since fixation, $e$ is the frequency at

185 which selection began, and $t_{d}$ is the time in generations since the two populations diverged. 

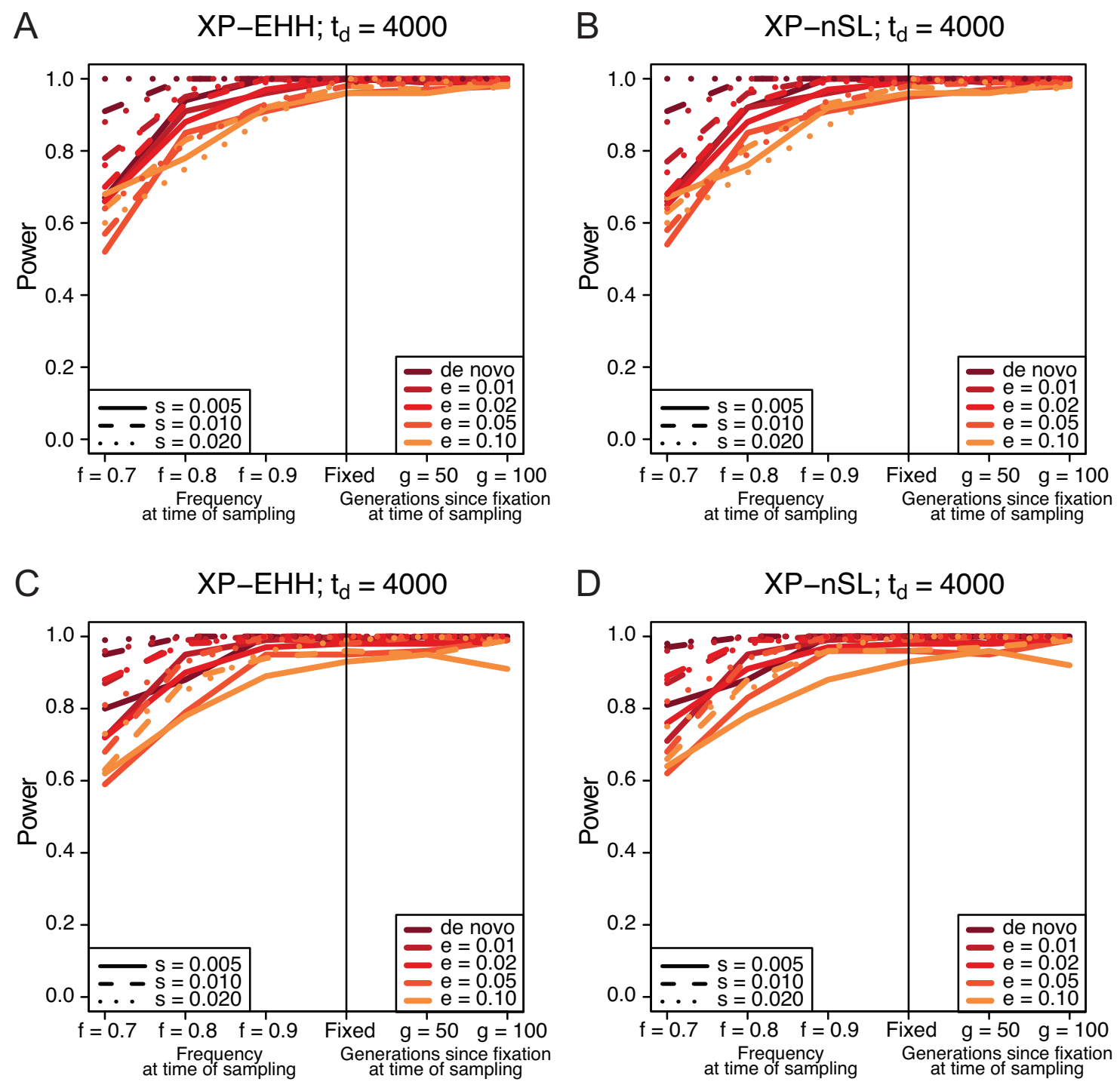

187 Figure 3. Power curves for unphased implementations of XP-EHH (A and C) and XP-nSL (B

188 and D) under demographic histories Demo 4 (A and B), and Demo 5 (C and D). $s$ is the

189 selection coefficient, $f$ is the frequency of the adaptive allele at time of sampling, $g$ is the

190 number of generations at time of sampling since fixation, $e$ is the frequency at which selection

191 began, and $t_{d}$ is the time in generations since the two populations diverged. 


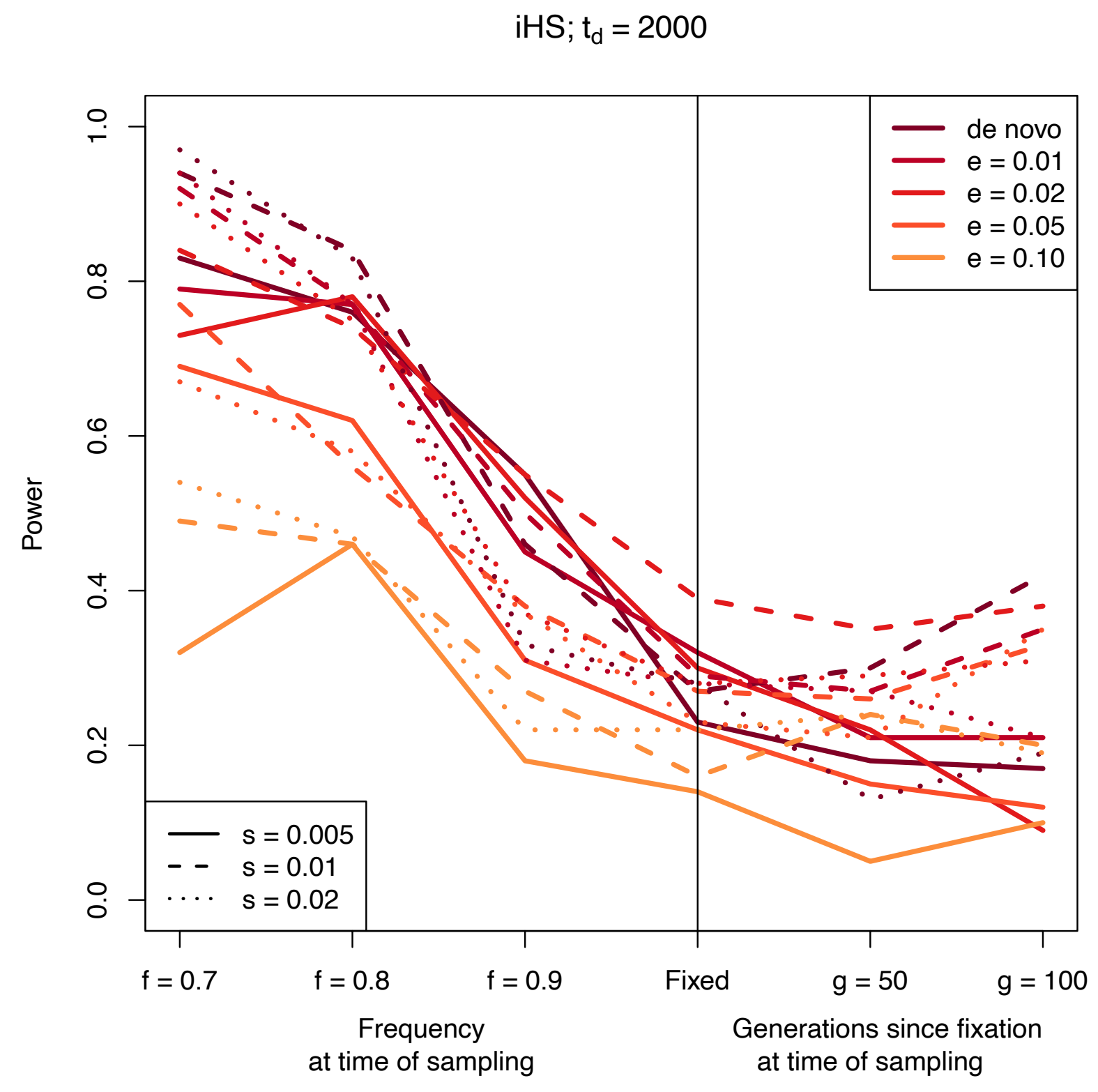

Figure S1. Demo $1 \mathrm{iHS} t_{d}=2000$ power curves. $s$ is the selection coefficient, $f$ is the

198 frequency of the adaptive allele at time of sampling, $g$ is the number of generations at time of

199 sampling since fixation, $e$ is the frequency at which selection began, and $t_{d}$ is the time in

200 generations since the two populations diverged. 


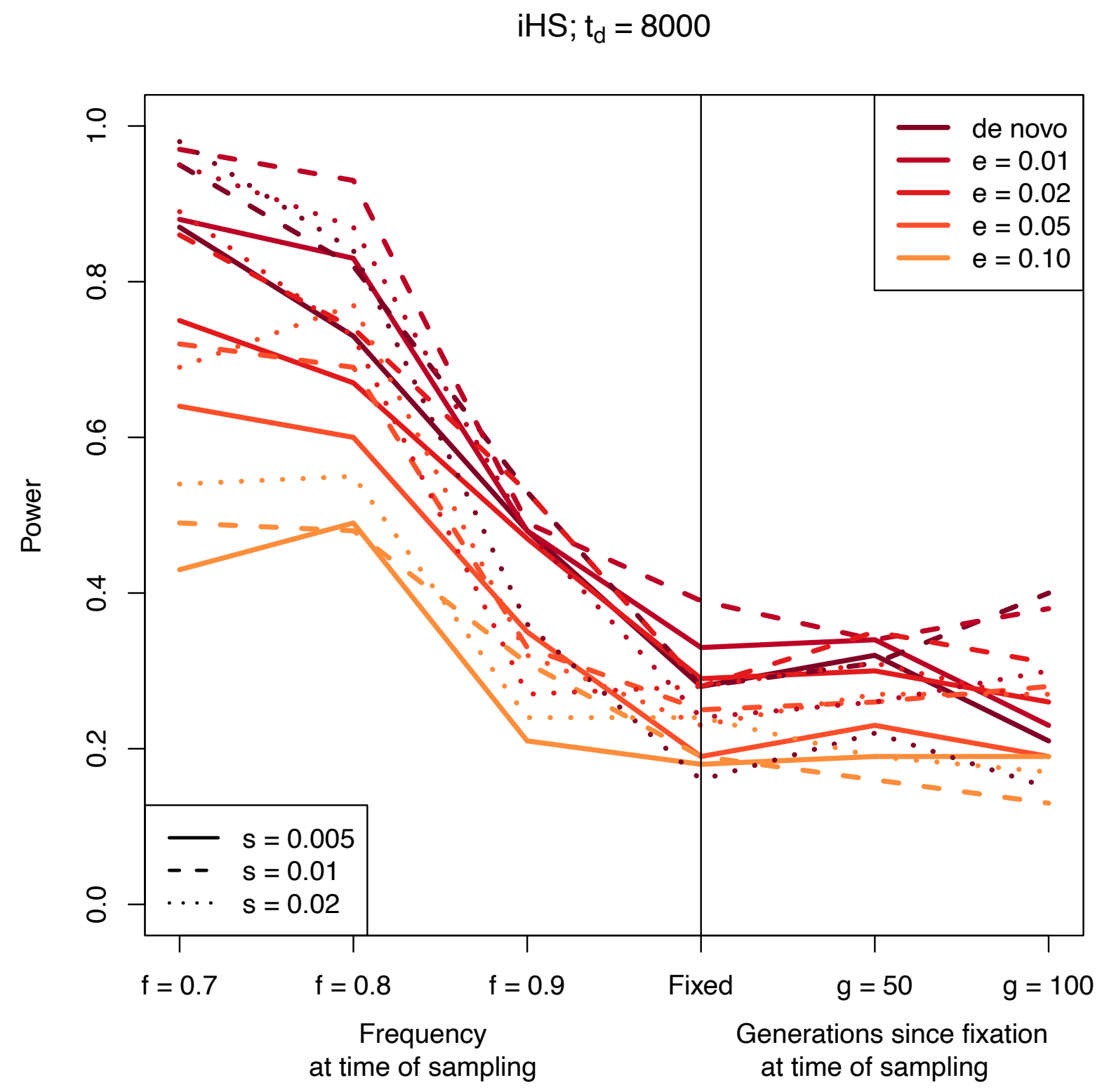

Figure S2. Demo $1 \mathrm{iHS} t_{d}=8000$ power curves. $s$ is the selection coefficient, $f$ is the

203 frequency of the adaptive allele at time of sampling, $g$ is the number of generations at time of

204 sampling since fixation, $e$ is the frequency at which selection began, and $t_{d}$ is the time in

205 generations since the two populations diverged. 


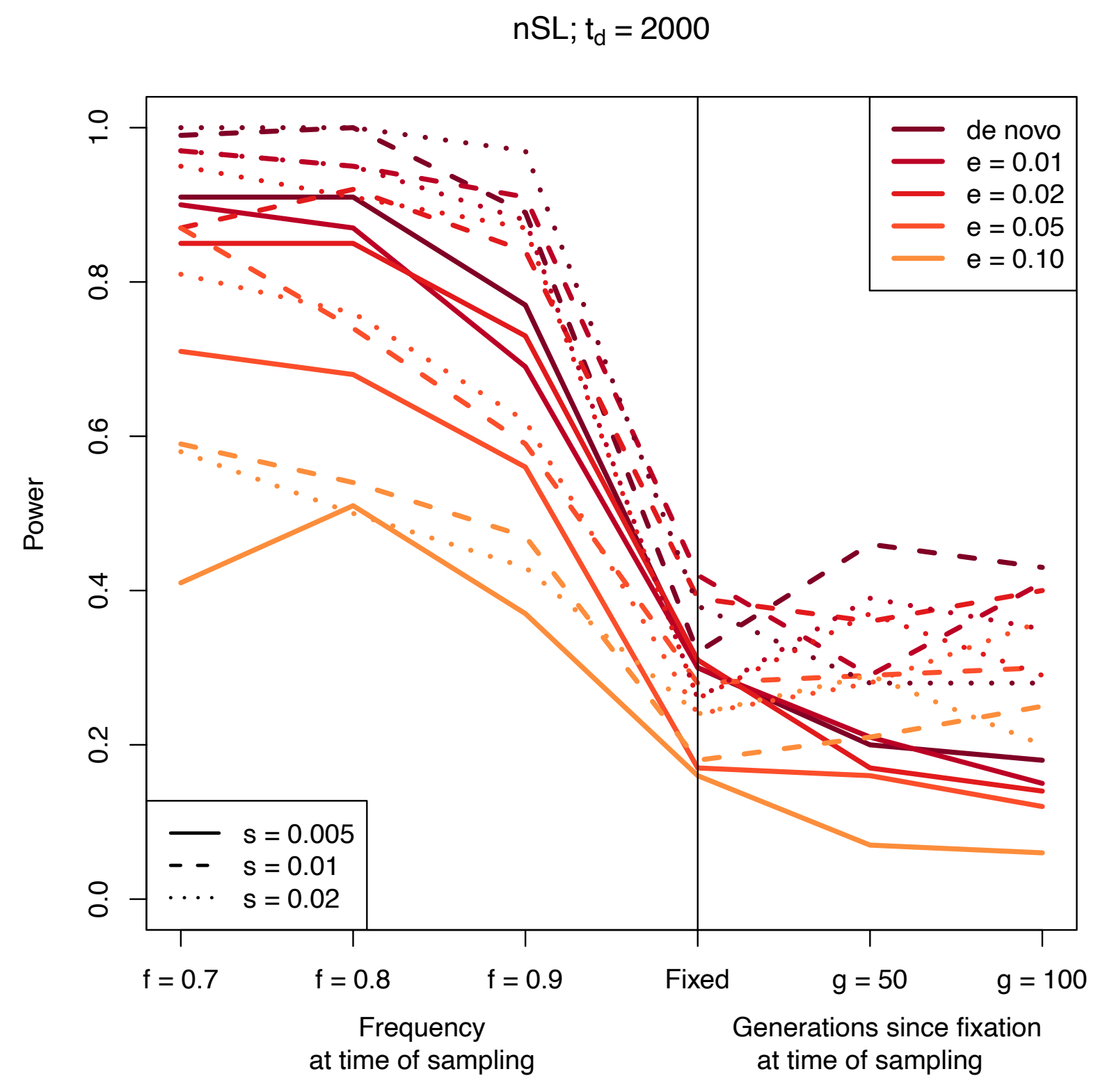

Figure S3. Demo $1 \mathrm{nSL} t_{d}=2000$ power curves. $s$ is the selection coefficient, $f$ is the

208 frequency of the adaptive allele at time of sampling, $g$ is the number of generations at time of

209 sampling since fixation, $e$ is the frequency at which selection began, and $t_{d}$ is the time in

210 generations since the two populations diverged. 


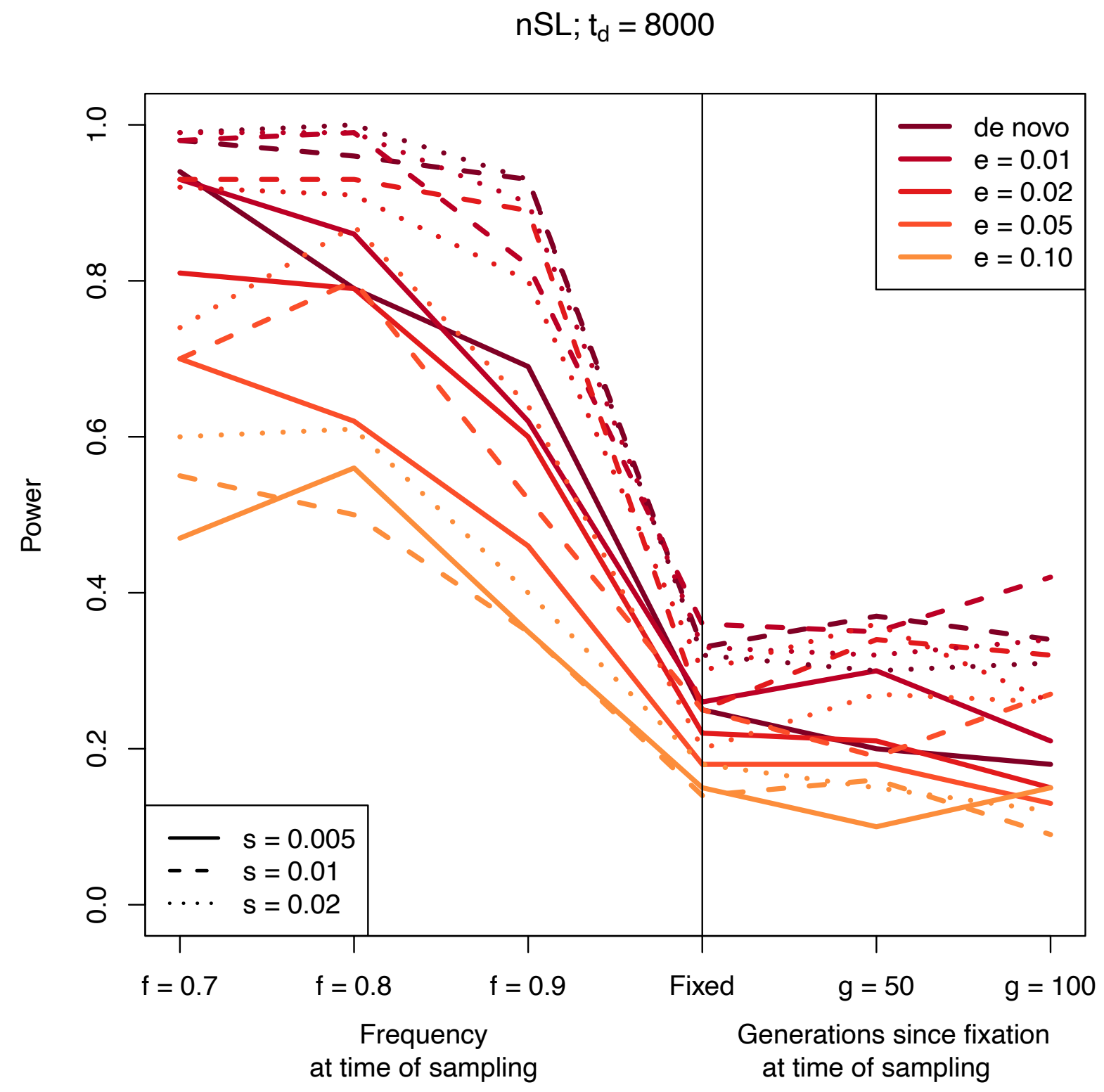

Figure S4. Demo $1 \mathrm{nSL} t_{d}=8000$ power curves. $s$ is the selection coefficient, $f$ is the

213 frequency of the adaptive allele at time of sampling, $g$ is the number of generations at time of

214 sampling since fixation, $e$ is the frequency at which selection began, and $t_{d}$ is the time in

215 generations since the two populations diverged. 


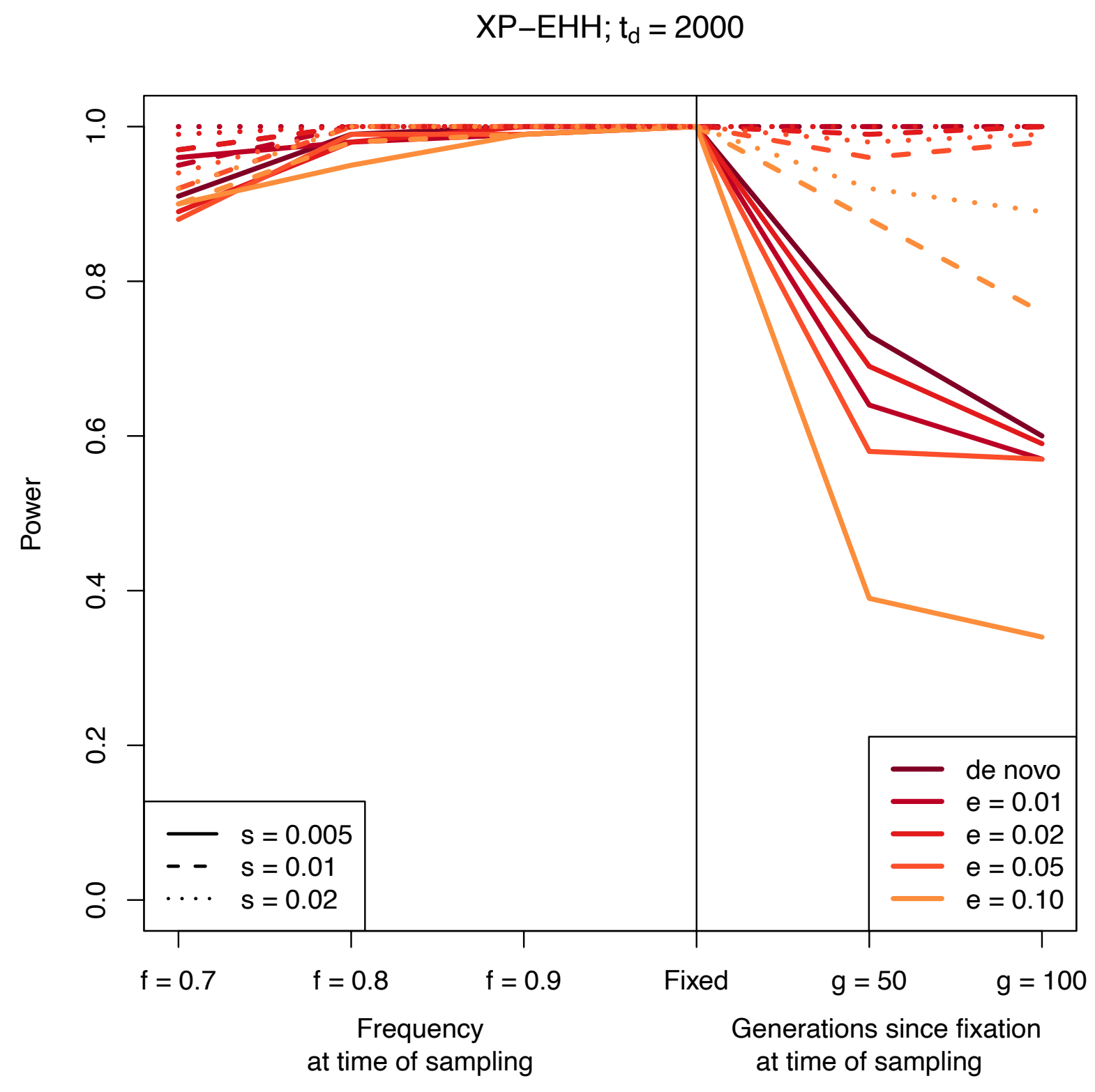

Figure S5. Demo $1 \mathrm{XP}-\mathrm{EHH} t_{d}=2000$ power curves. $s$ is the selection coefficient, $f$ is the

218 frequency of the adaptive allele at time of sampling, $g$ is the number of generations at time of

219 sampling since fixation, $e$ is the frequency at which selection began, and $t_{d}$ is the time in

220 generations since the two populations diverged. 


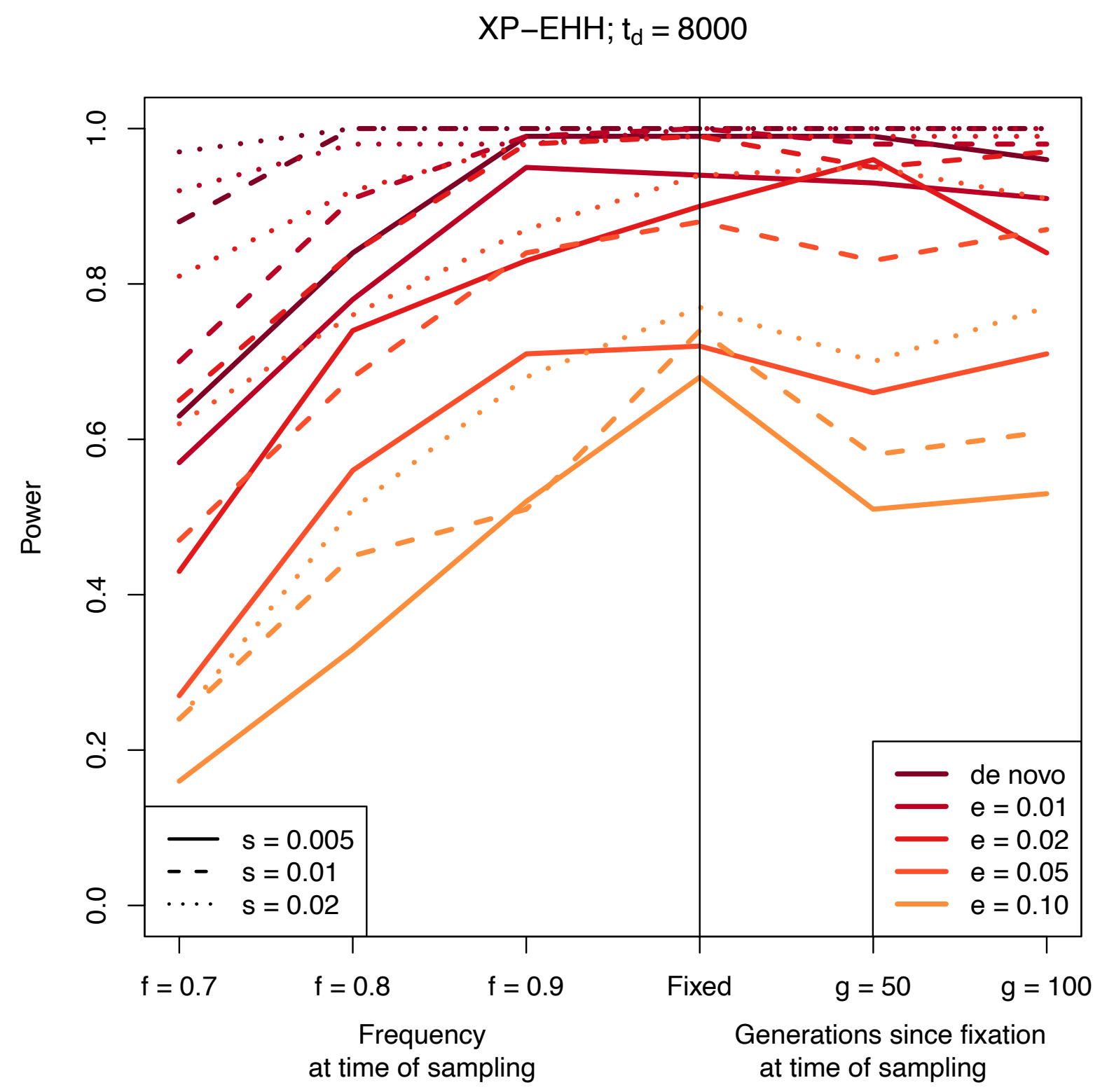

Figure S6. Demo $1 \mathrm{XP}-\mathrm{EHH} t_{d}=8000$ power curves. $s$ is the selection coefficient, $f$ is the

223 frequency of the adaptive allele at time of sampling, $g$ is the number of generations at time of

224 sampling since fixation, $e$ is the frequency at which selection began, and $t_{d}$ is the time in

225 generations since the two populations diverged. 


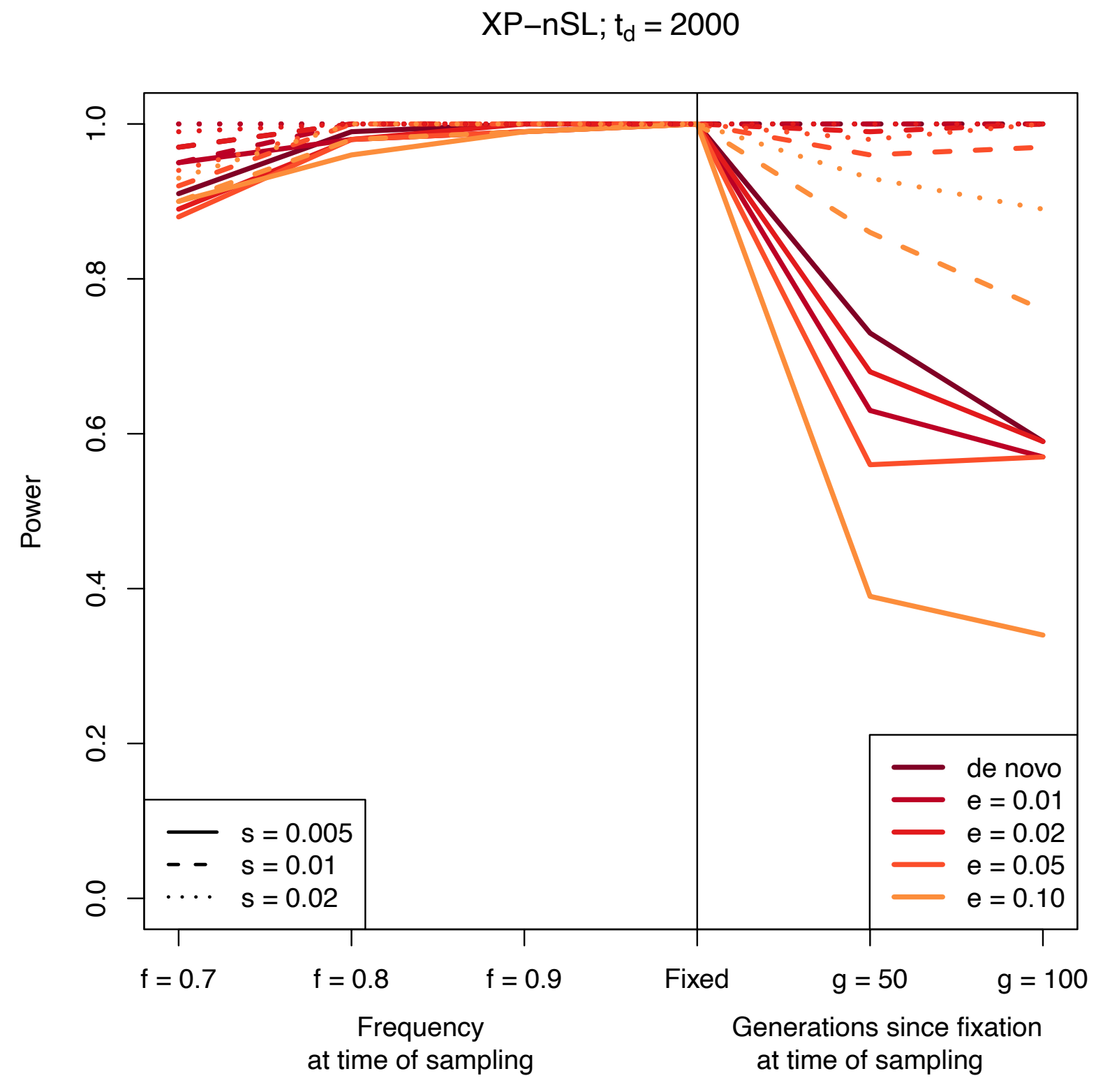

227 Figure S7. Demo 1 XP-nSL $t_{d}=2000$ power curves. $s$ is the selection coefficient, $f$ is the

228 frequency of the adaptive allele at time of sampling, $g$ is the number of generations at time of

229 sampling since fixation, $e$ is the frequency at which selection began, and $t_{d}$ is the time in

230 generations since the two populations diverged. 


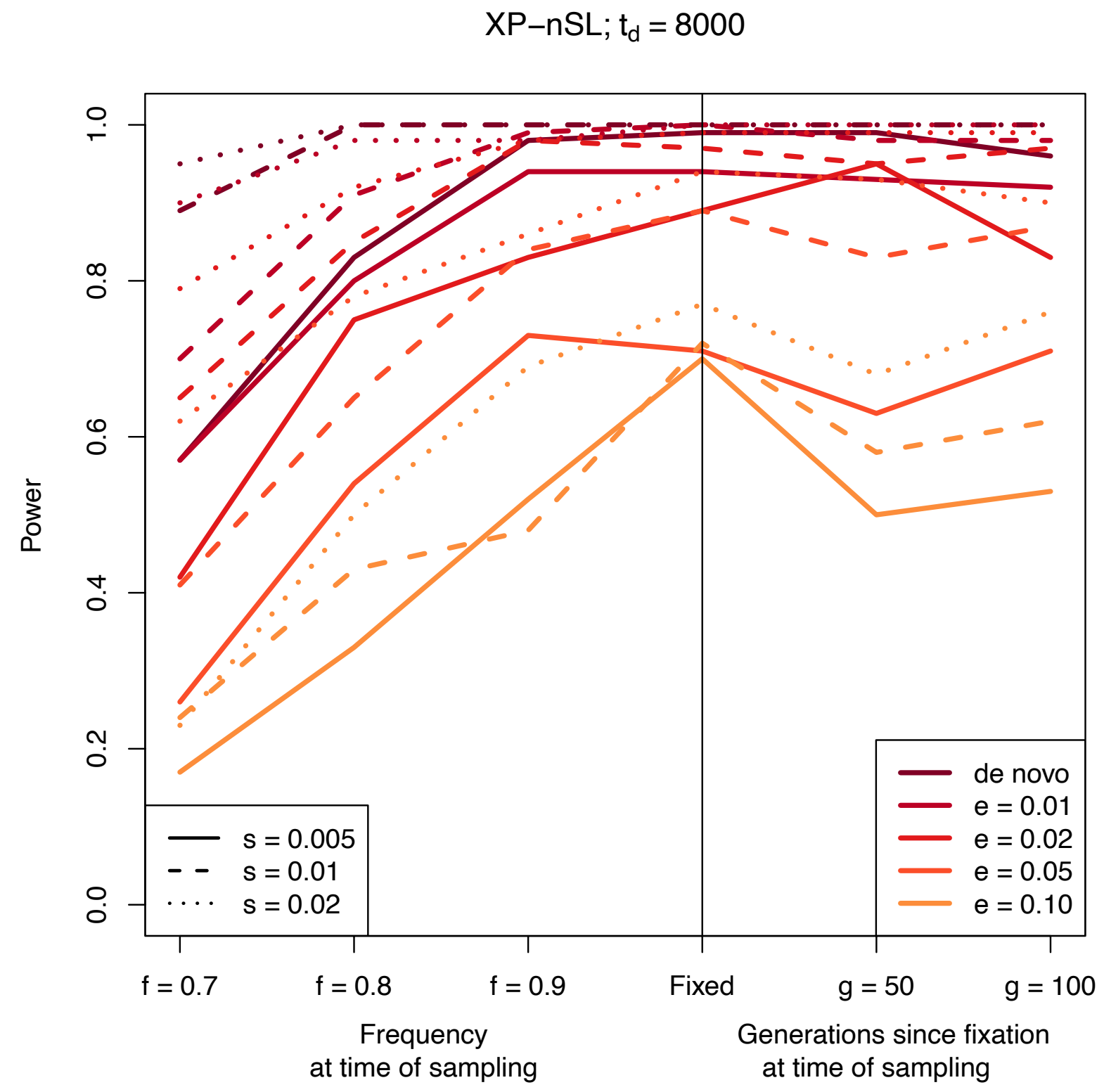

Figure S8. Demo 1 XP-nSL $t_{d}=8000$ power curves. $s$ is the selection coefficient, $f$ is the

233 frequency of the adaptive allele at time of sampling, $g$ is the number of generations at time of

234 sampling since fixation, $e$ is the frequency at which selection began, and $t_{d}$ is the time in

235 generations since the two populations diverged. 


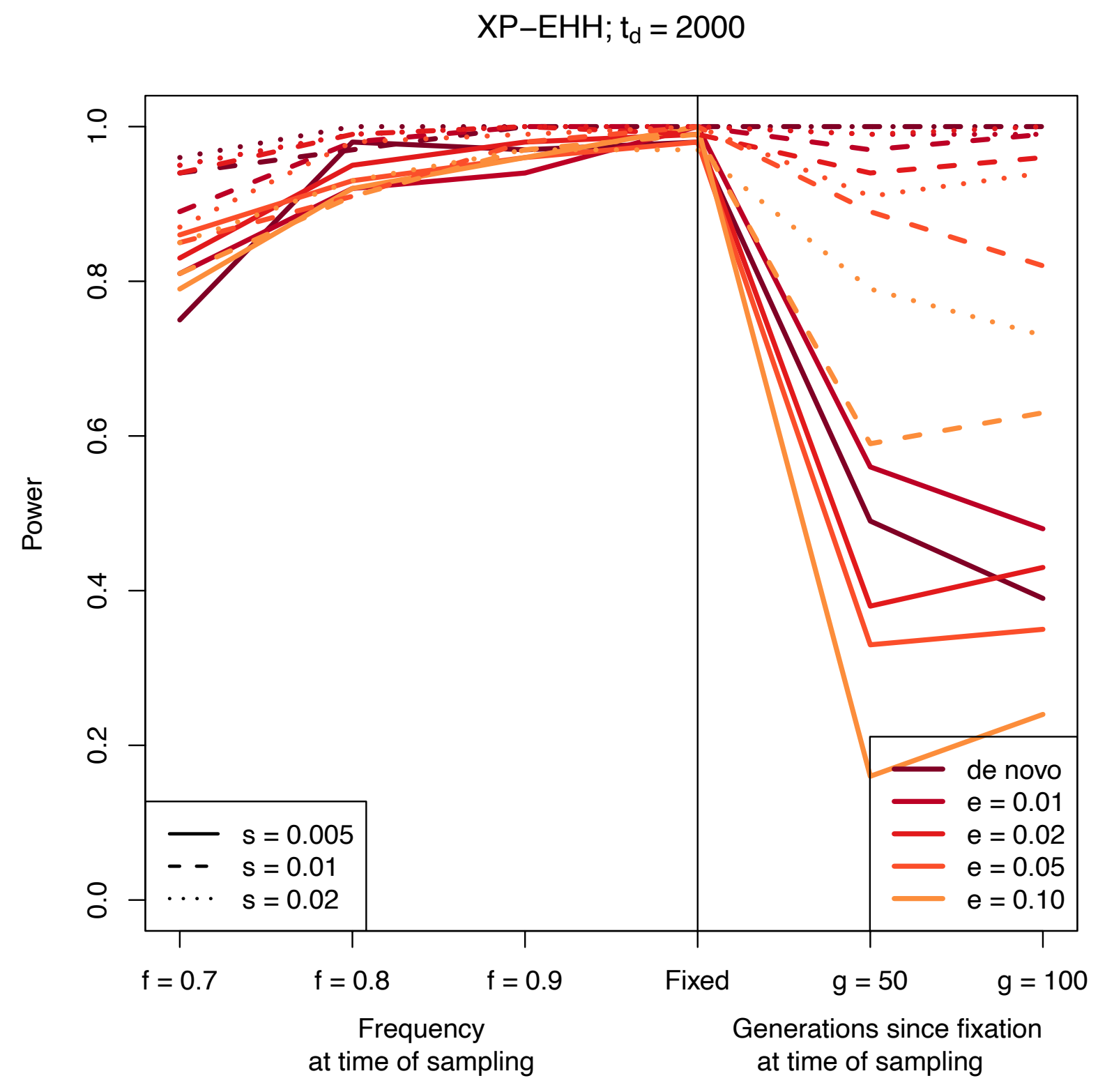

Figure S9. Demo $2 \mathrm{XP}-\mathrm{EHH} t_{d}=2000$ power curves. $s$ is the selection coefficient, $f$ is the

238 frequency of the adaptive allele at time of sampling, $g$ is the number of generations at time of

239 sampling since fixation, $e$ is the frequency at which selection began, and $t_{d}$ is the time in

240 generations since the two populations diverged. 




Figure S10. Demo 2 XP-EHH $t_{d}=8000$ power curves. $s$ is the selection coefficient, $f$ is the

243 frequency of the adaptive allele at time of sampling, $g$ is the number of generations at time of

244 sampling since fixation, $e$ is the frequency at which selection began, and $t_{d}$ is the time in

245 generations since the two populations diverged. 


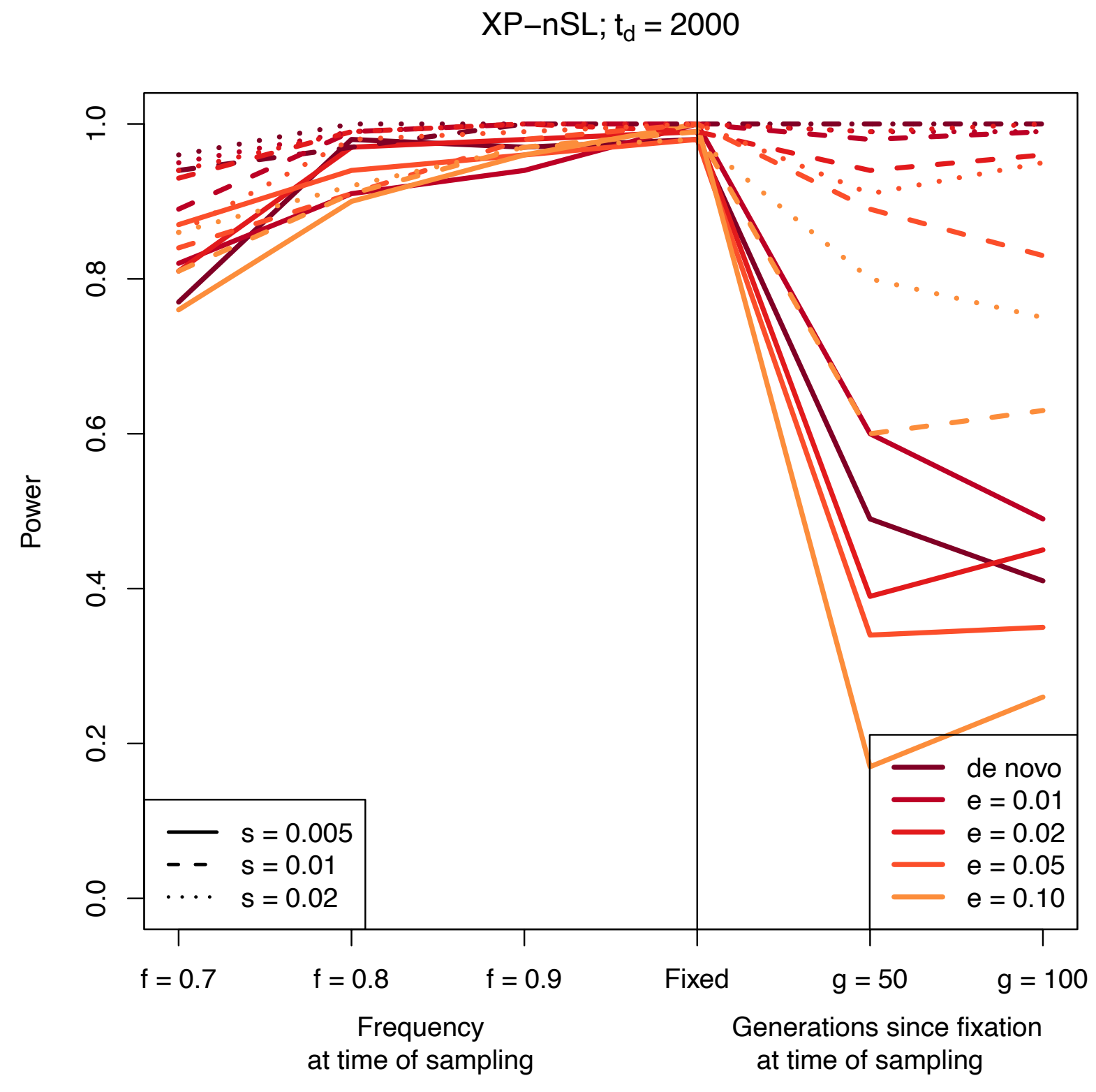

Figure S11. Demo 2 XP-nSL $t_{d}=2000$ power curves. $s$ is the selection coefficient, $f$ is the

248 frequency of the adaptive allele at time of sampling, $g$ is the number of generations at time of

249 sampling since fixation, $e$ is the frequency at which selection began, and $t_{d}$ is the time in

250 generations since the two populations diverged. 


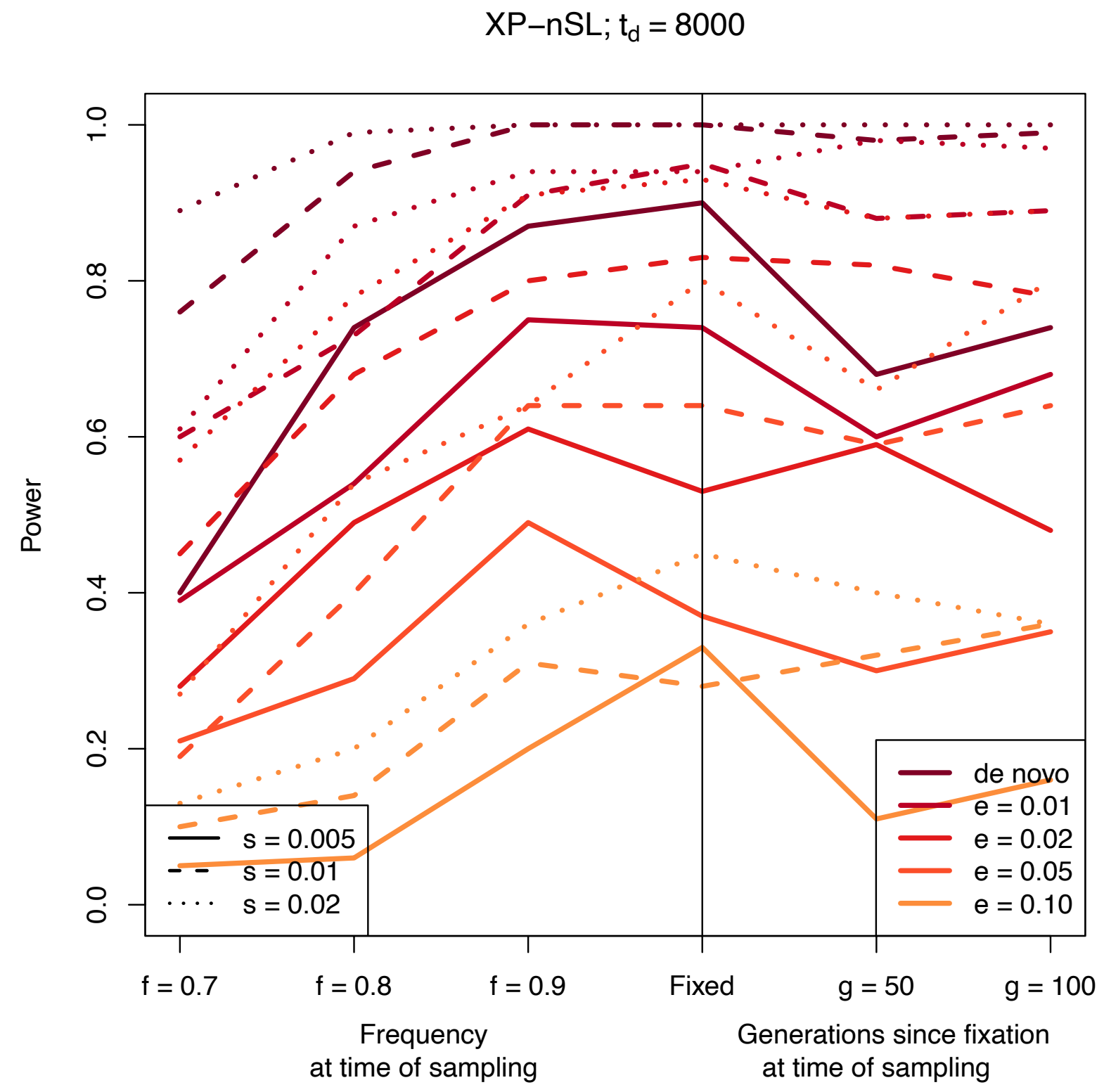

Figure S12. Demo 2 XP-nSL $t_{d}=8000$ power curves. $s$ is the selection coefficient, $f$ is the

253 frequency of the adaptive allele at time of sampling, $g$ is the number of generations at time of

254 sampling since fixation, $e$ is the frequency at which selection began, and $t_{d}$ is the time in

255 generations since the two populations diverged. 


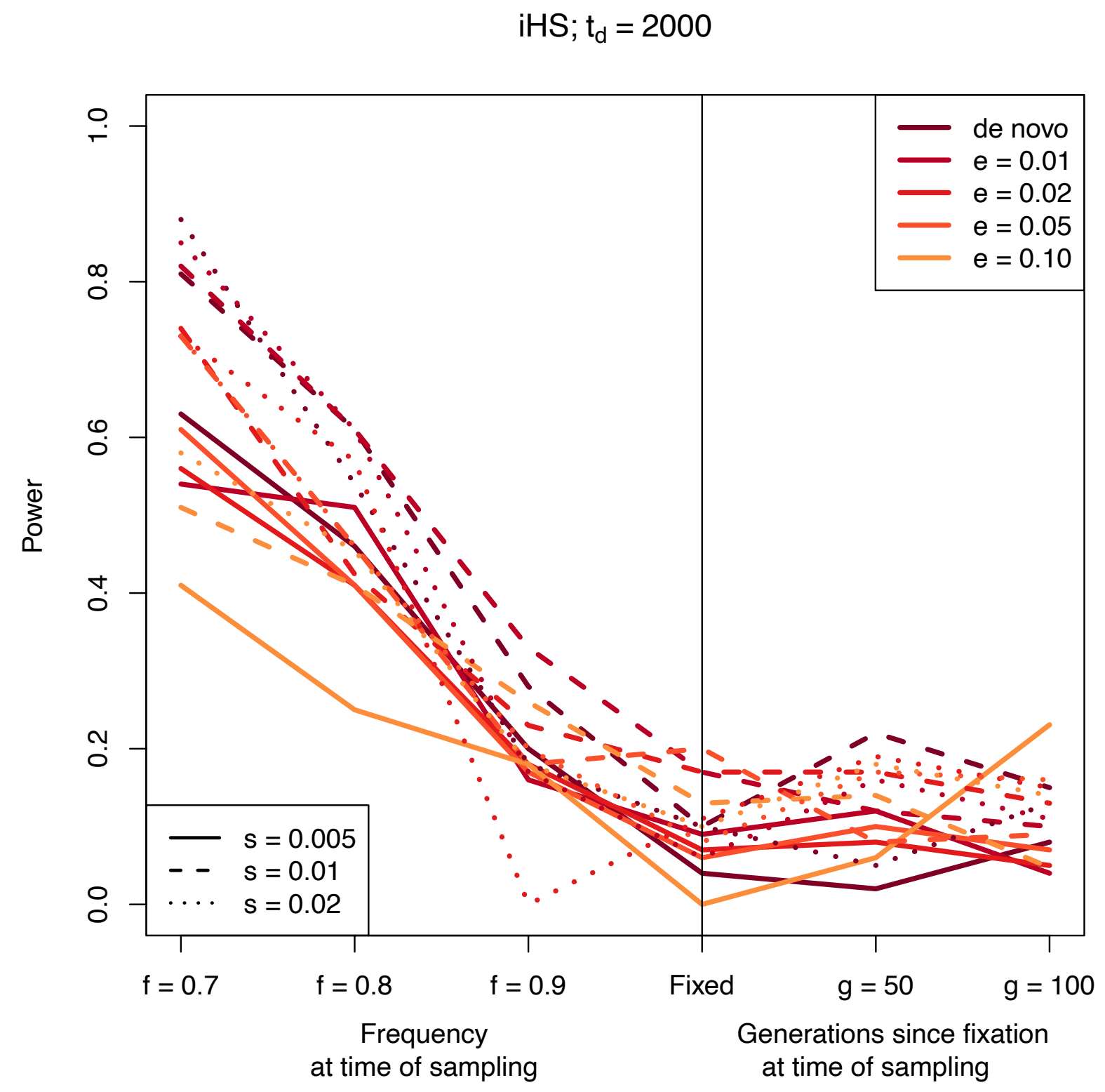

Figure S13. Demo 3 iHS $t_{d}=2000$ power curves. $s$ is the selection coefficient, $f$ is the

258 frequency of the adaptive allele at time of sampling, $g$ is the number of generations at time of

259 sampling since fixation, $e$ is the frequency at which selection began, and $t_{d}$ is the time in

260 generations since the two populations diverged. 


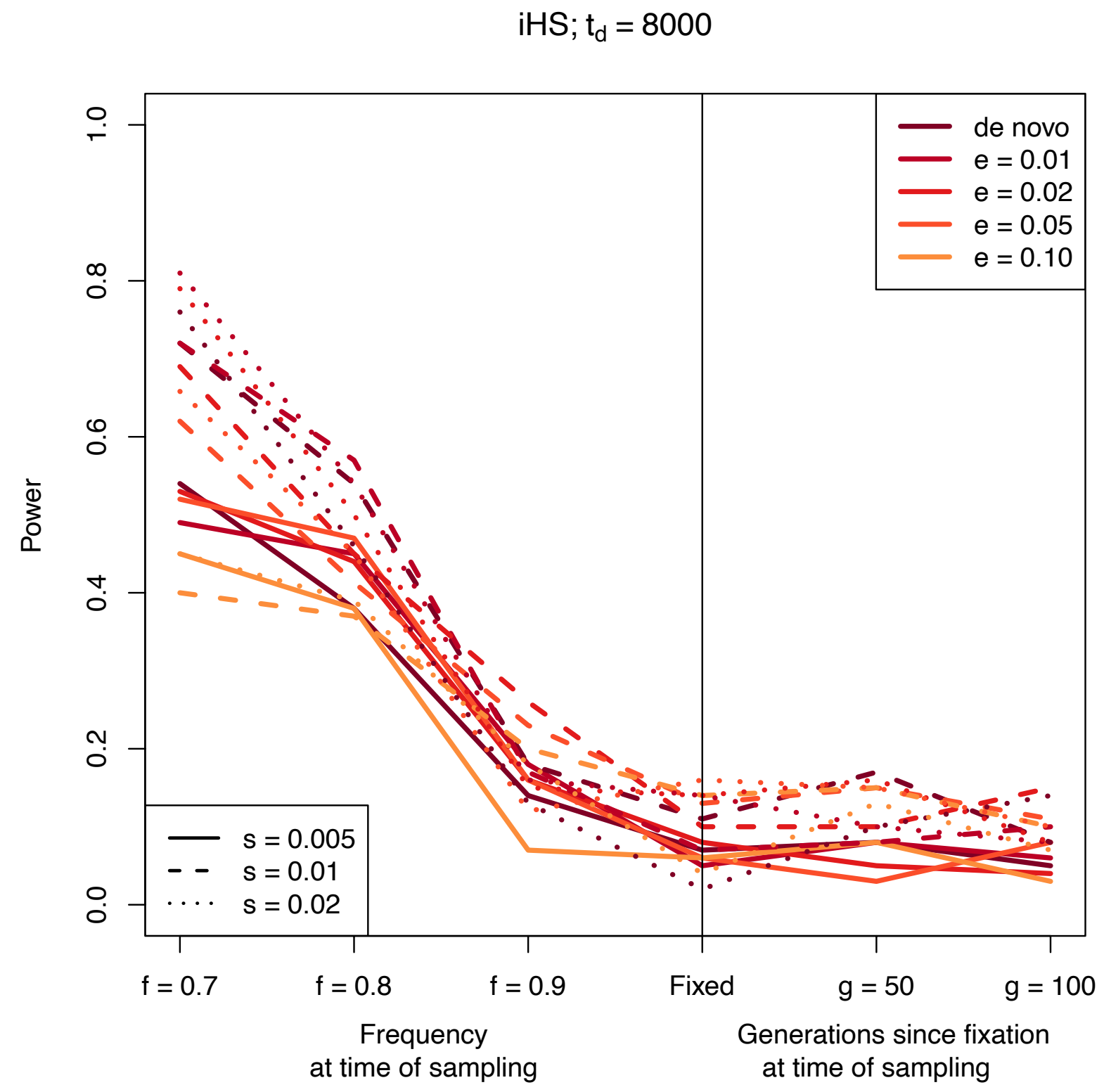

Figure S14. Demo $3 \mathrm{iHS} t_{d}=8000$ power curves. $s$ is the selection coefficient, $f$ is the

263 frequency of the adaptive allele at time of sampling, $g$ is the number of generations at time of

264 sampling since fixation, $e$ is the frequency at which selection began, and $t_{d}$ is the time in

265 generations since the two populations diverged. 


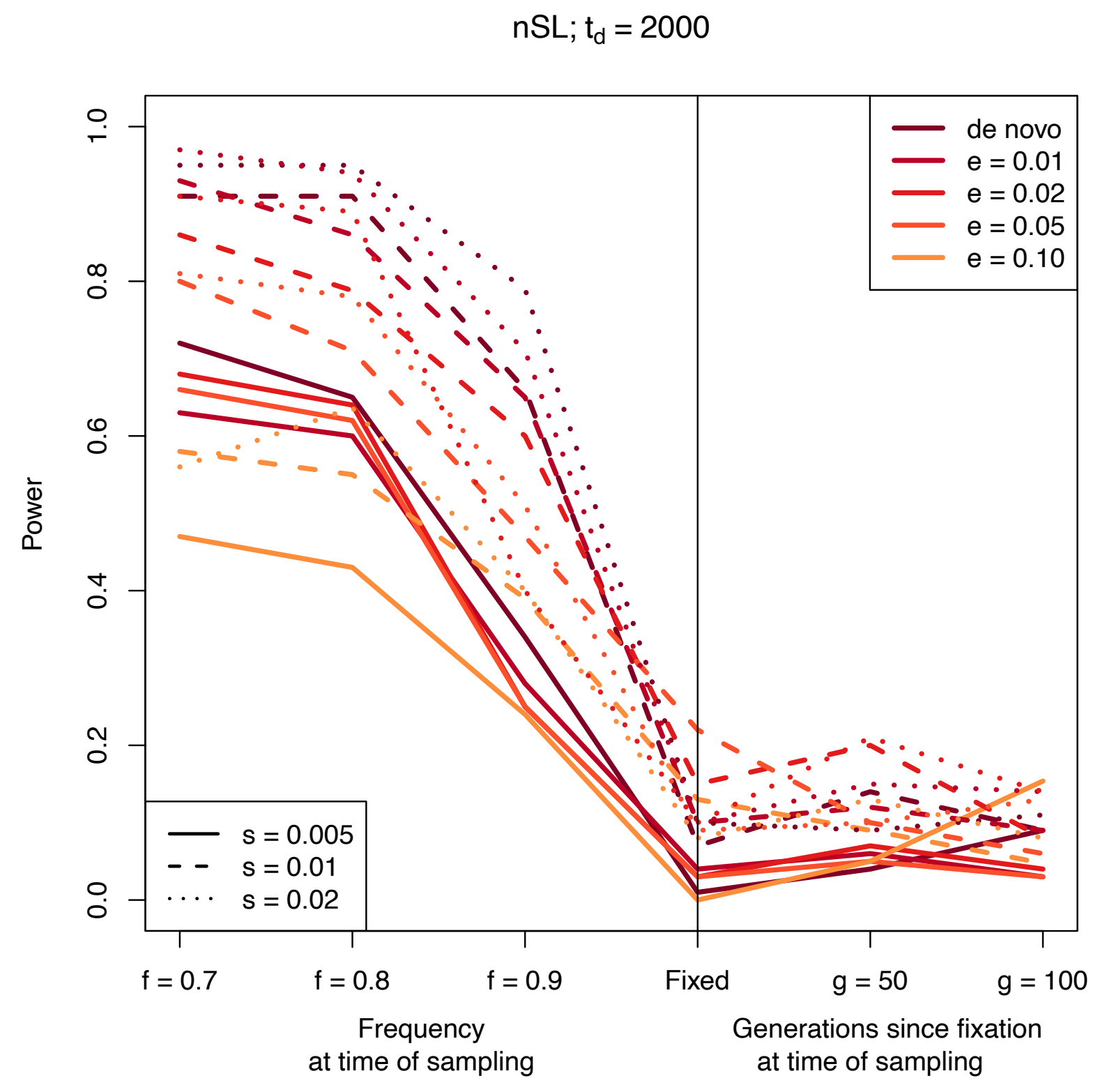

Figure S15. Demo $3 \mathrm{nSL} t_{d}=2000$ power curves. $s$ is the selection coefficient, $f$ is the

268 frequency of the adaptive allele at time of sampling, $g$ is the number of generations at time of

269 sampling since fixation, $e$ is the frequency at which selection began, and $t_{d}$ is the time in

270 generations since the two populations diverged. 


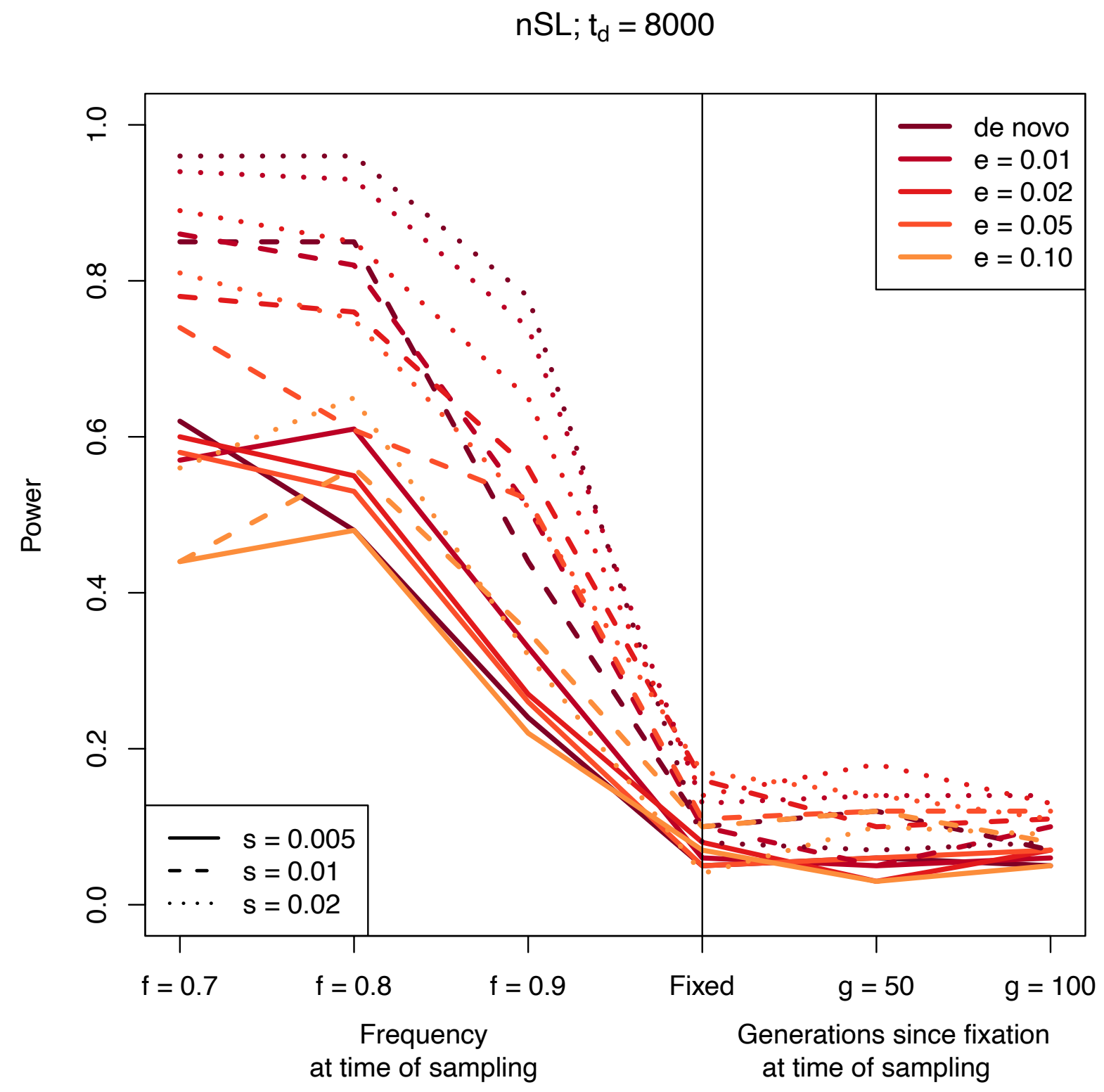

Figure S16. Demo $3 \mathrm{nSL} t_{d}=8000$ power curves. $s$ is the selection coefficient, $f$ is the

273 frequency of the adaptive allele at time of sampling, $g$ is the number of generations at time of

274 sampling since fixation, $e$ is the frequency at which selection began, and $t_{d}$ is the time in

275 generations since the two populations diverged. 


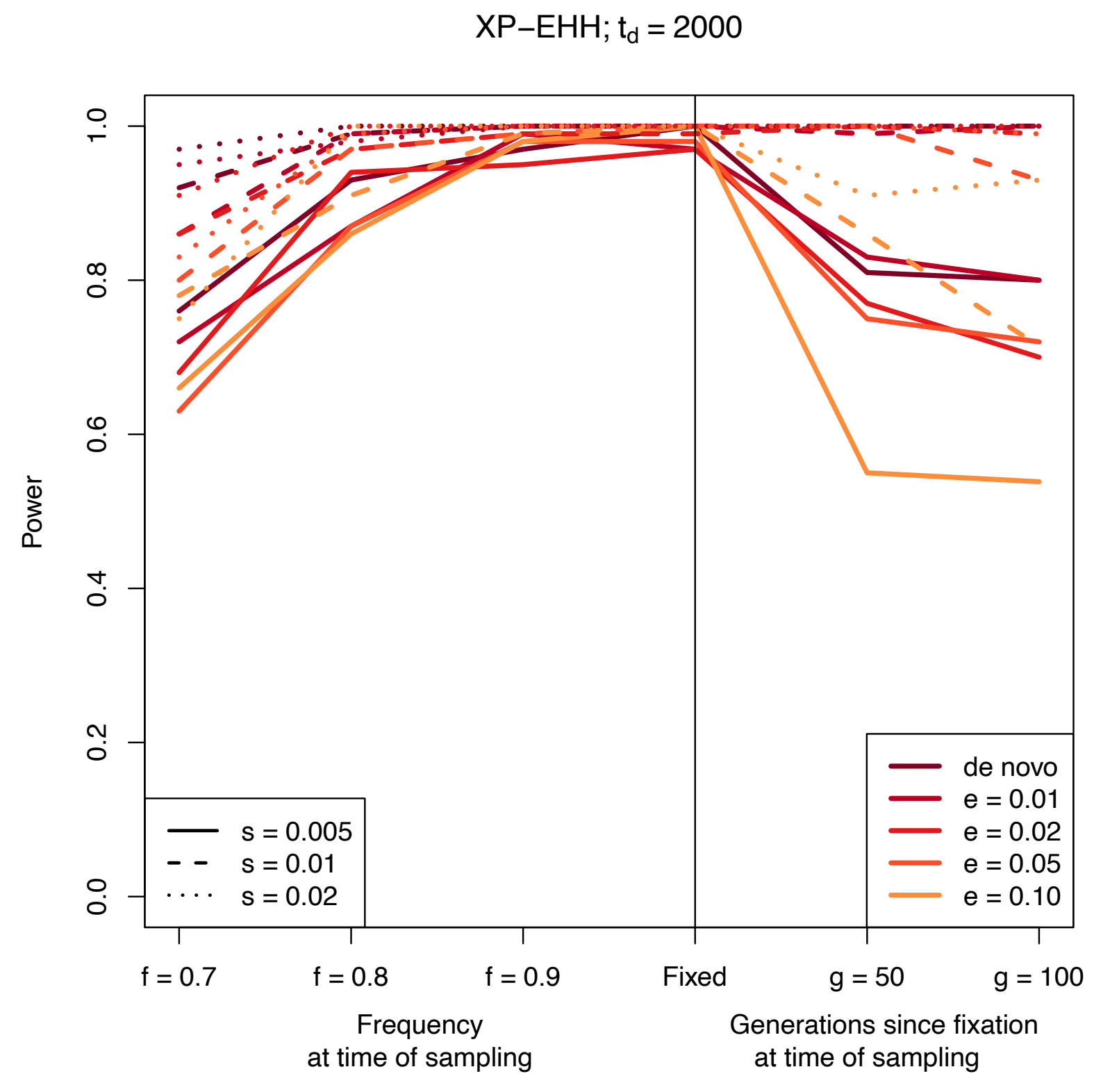

277 Figure S17. Demo $3 \mathrm{XP}-\mathrm{EHH} t_{d}=2000$ power curves. $s$ is the selection coefficient, $f$ is the

278 frequency of the adaptive allele at time of sampling, $g$ is the number of generations at time of

279 sampling since fixation, $e$ is the frequency at which selection began, and $t_{d}$ is the time in

280 generations since the two populations diverged. 


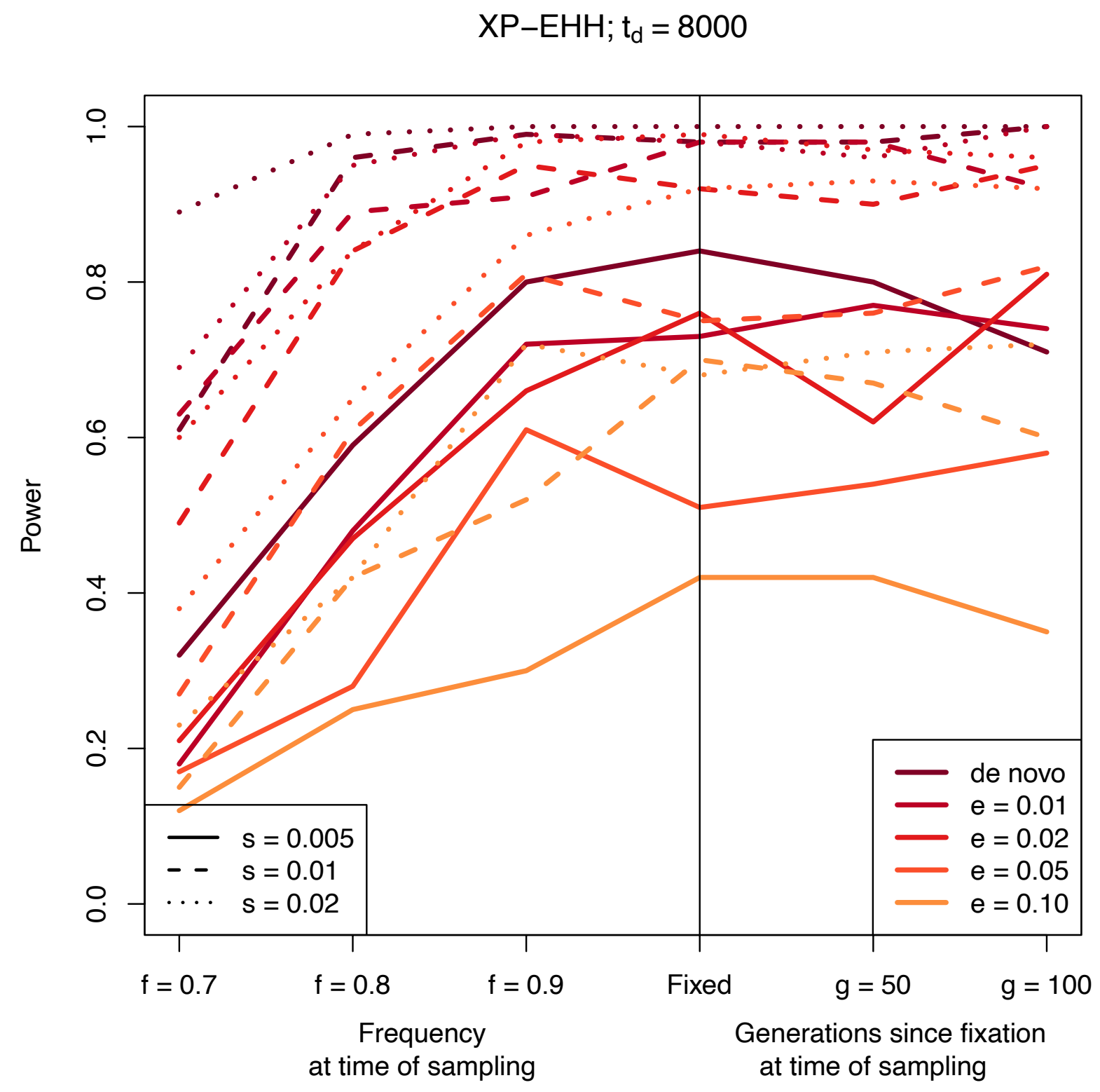

Figure S18. Demo $3 \mathrm{XP}-\mathrm{EHH} t_{d}=8000$ power curves. $s$ is the selection coefficient, $f$ is the

283 frequency of the adaptive allele at time of sampling, $g$ is the number of generations at time of

284 sampling since fixation, $e$ is the frequency at which selection began, and $t_{d}$ is the time in

285 generations since the two populations diverged. 


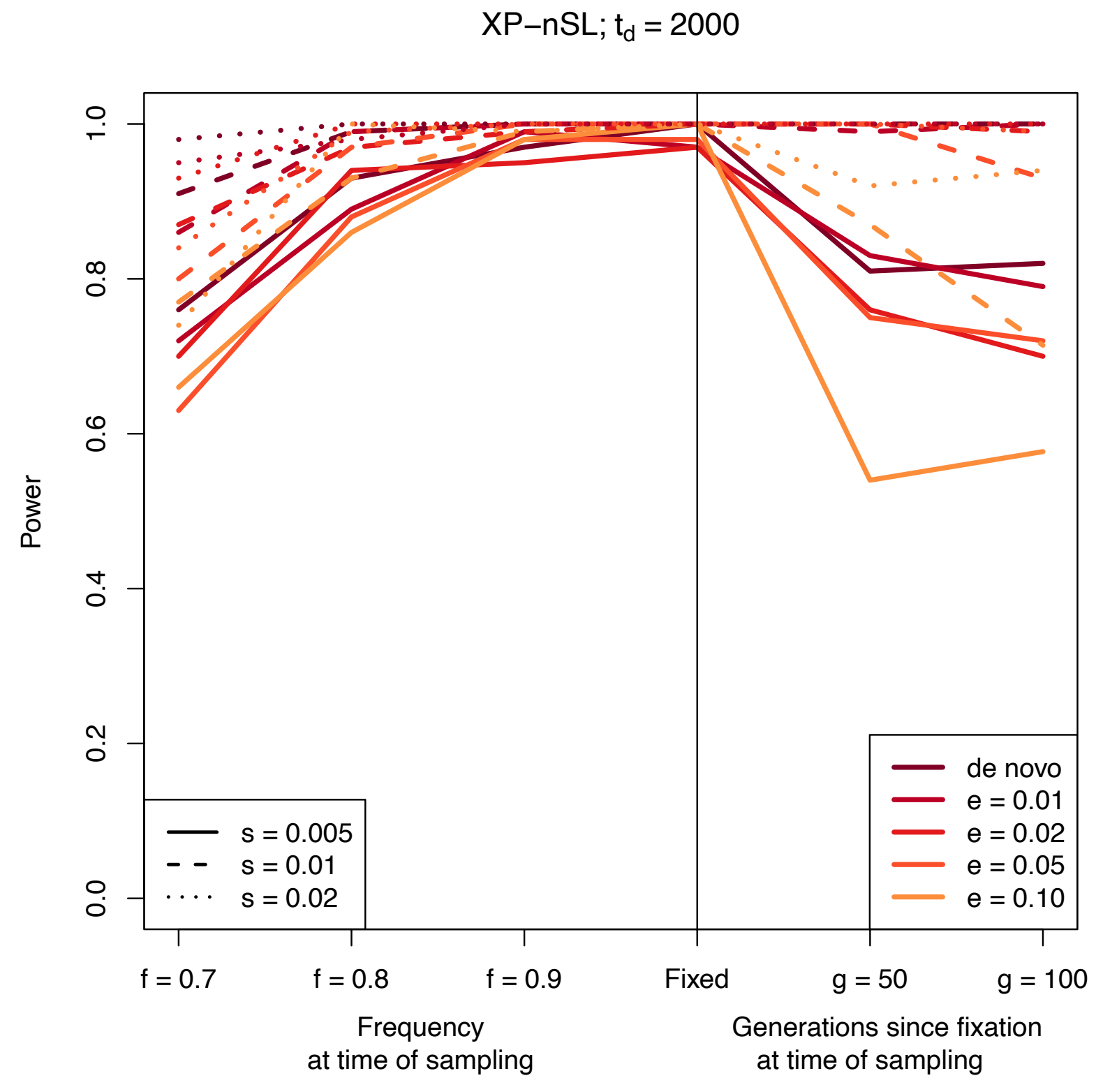

Figure S19. Demo 3 XP-nSL $t_{d}=2000$ power curves. $s$ is the selection coefficient, $f$ is the

288 frequency of the adaptive allele at time of sampling, $g$ is the number of generations at time of

289 sampling since fixation, $e$ is the frequency at which selection began, and $t_{d}$ is the time in

290 generations since the two populations diverged. 


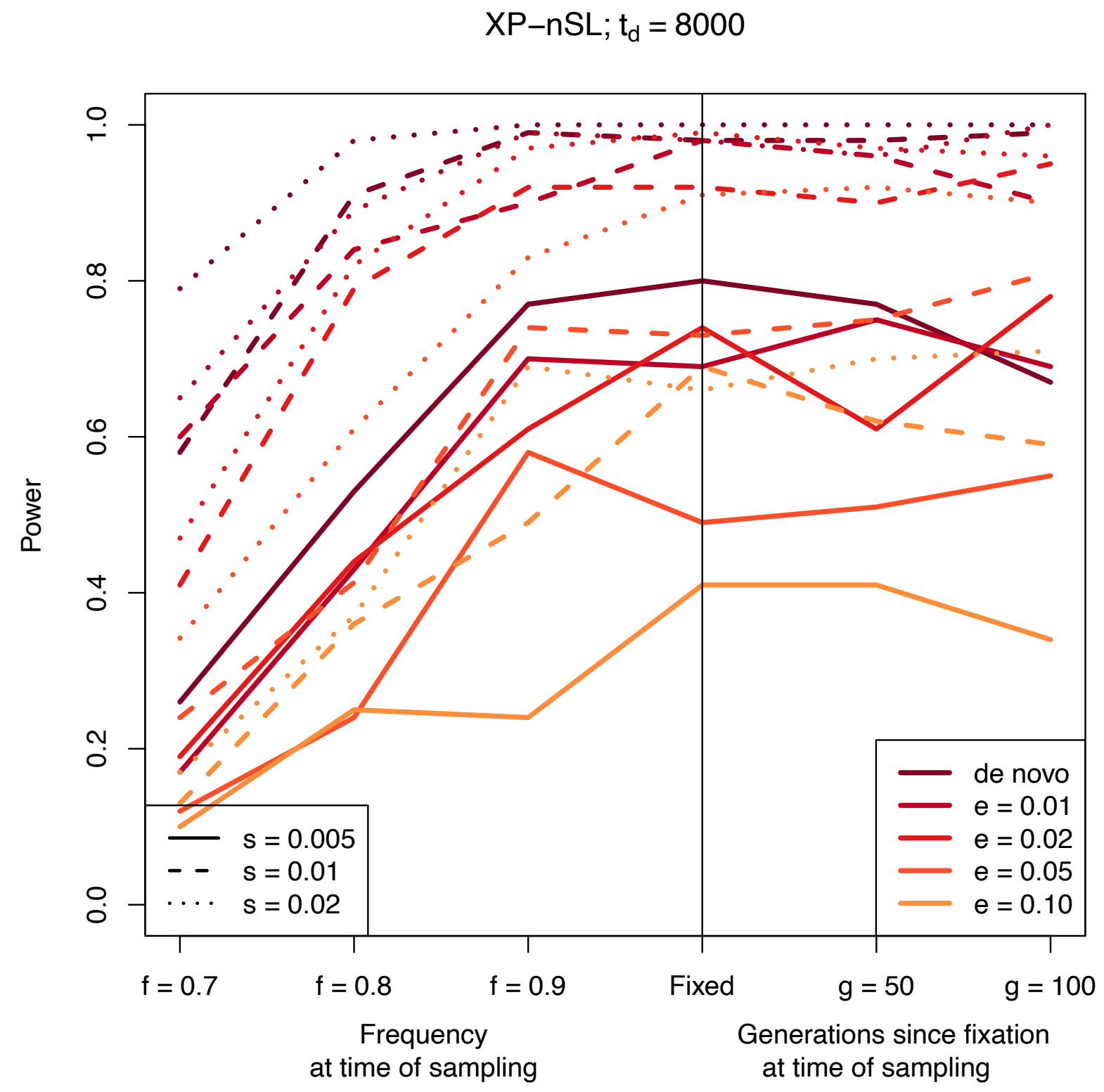

Figure S20. Demo 3 XP-nSL $t_{d}=8000$ power curves. $s$ is the selection coefficient, $f$ is the

293 frequency of the adaptive allele at time of sampling, $g$ is the number of generations at time of

294 sampling since fixation, $e$ is the frequency at which selection began, and $t_{d}$ is the time in

295 generations since the two populations diverged. 


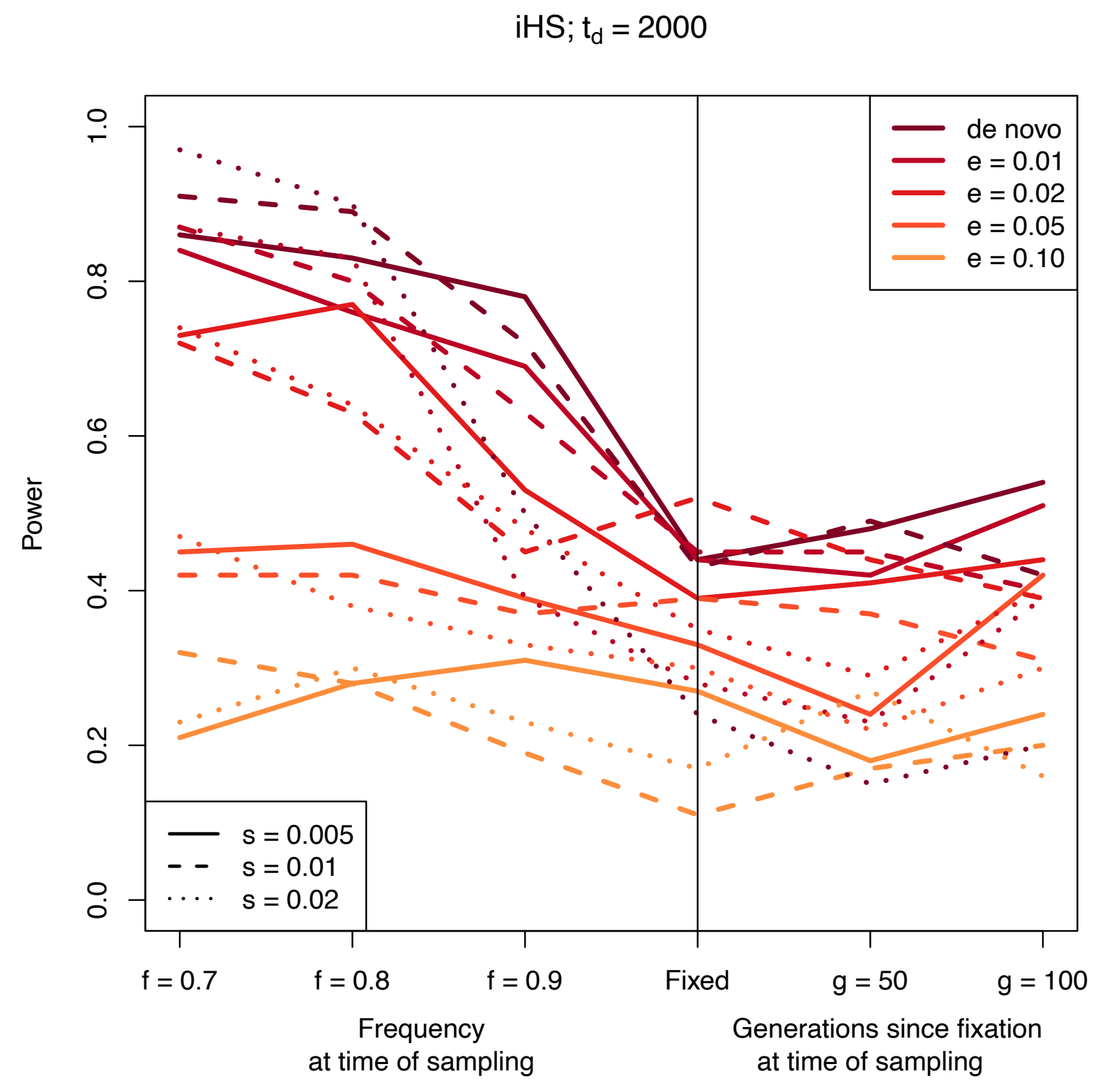

Figure S21. Demo 4 iHS $t_{d}=2000$ power curves. $s$ is the selection coefficient, $f$ is the

298 frequency of the adaptive allele at time of sampling, $g$ is the number of generations at time of

299 sampling since fixation, $e$ is the frequency at which selection began, and $t_{d}$ is the time in

300 generations since the two populations diverged. 


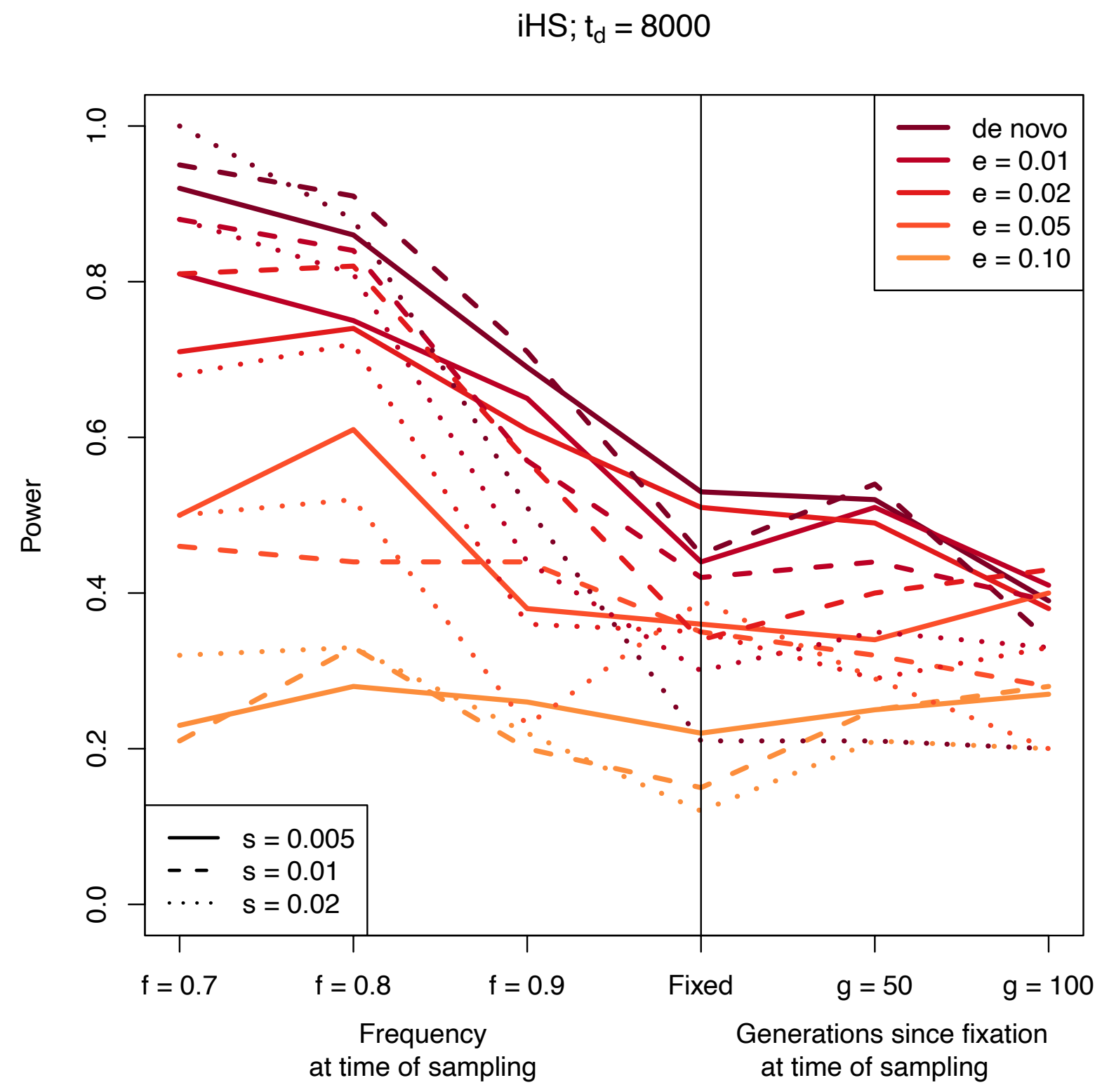

Figure S22. Demo 4 iHS $t_{d}=8000$ power curves. $s$ is the selection coefficient, $f$ is the

303 frequency of the adaptive allele at time of sampling, $g$ is the number of generations at time of

304 sampling since fixation, $e$ is the frequency at which selection began, and $t_{d}$ is the time in

305 generations since the two populations diverged. 


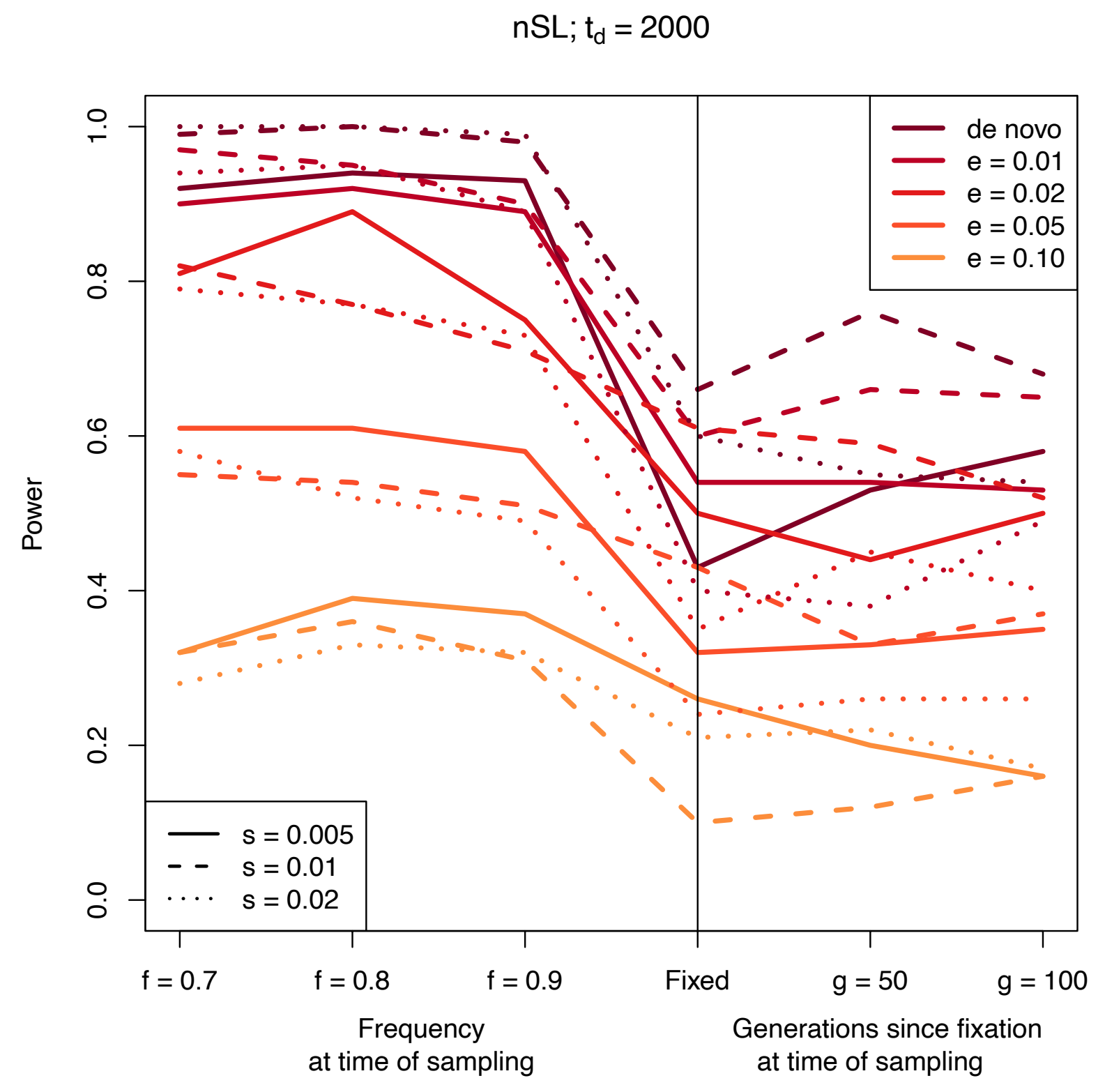

Figure S23. Demo $4 \mathrm{nSL} t_{d}=2000$ power curves. $s$ is the selection coefficient, $f$ is the

308 frequency of the adaptive allele at time of sampling, $g$ is the number of generations at time of

309 sampling since fixation, $e$ is the frequency at which selection began, and $t_{d}$ is the time in

310 generations since the two populations diverged. 




Figure S24. Demo $4 \mathrm{nSL} t_{d}=8000$ power curves. $s$ is the selection coefficient, $f$ is the

313 frequency of the adaptive allele at time of sampling, $g$ is the number of generations at time of

314 sampling since fixation, $e$ is the frequency at which selection began, and $t_{d}$ is the time in

315 generations since the two populations diverged. 


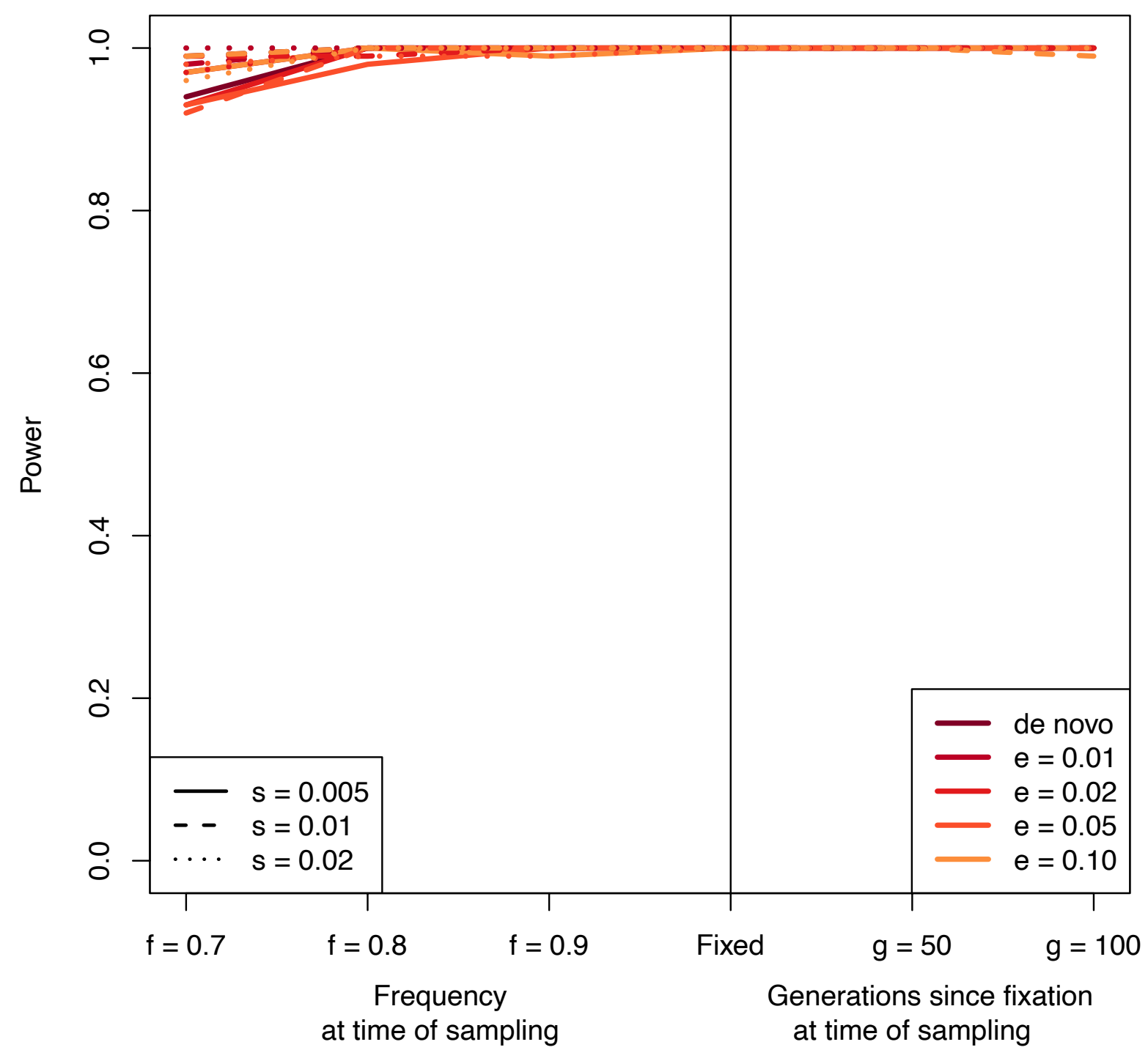

317 Figure S25. Demo $4 \mathrm{XP}$-EHH $t_{d}=2000$ power curves. $s$ is the selection coefficient, $f$ is the

318 frequency of the adaptive allele at time of sampling, $g$ is the number of generations at time of

319 sampling since fixation, $e$ is the frequency at which selection began, and $t_{d}$ is the time in

320 generations since the two populations diverged. 


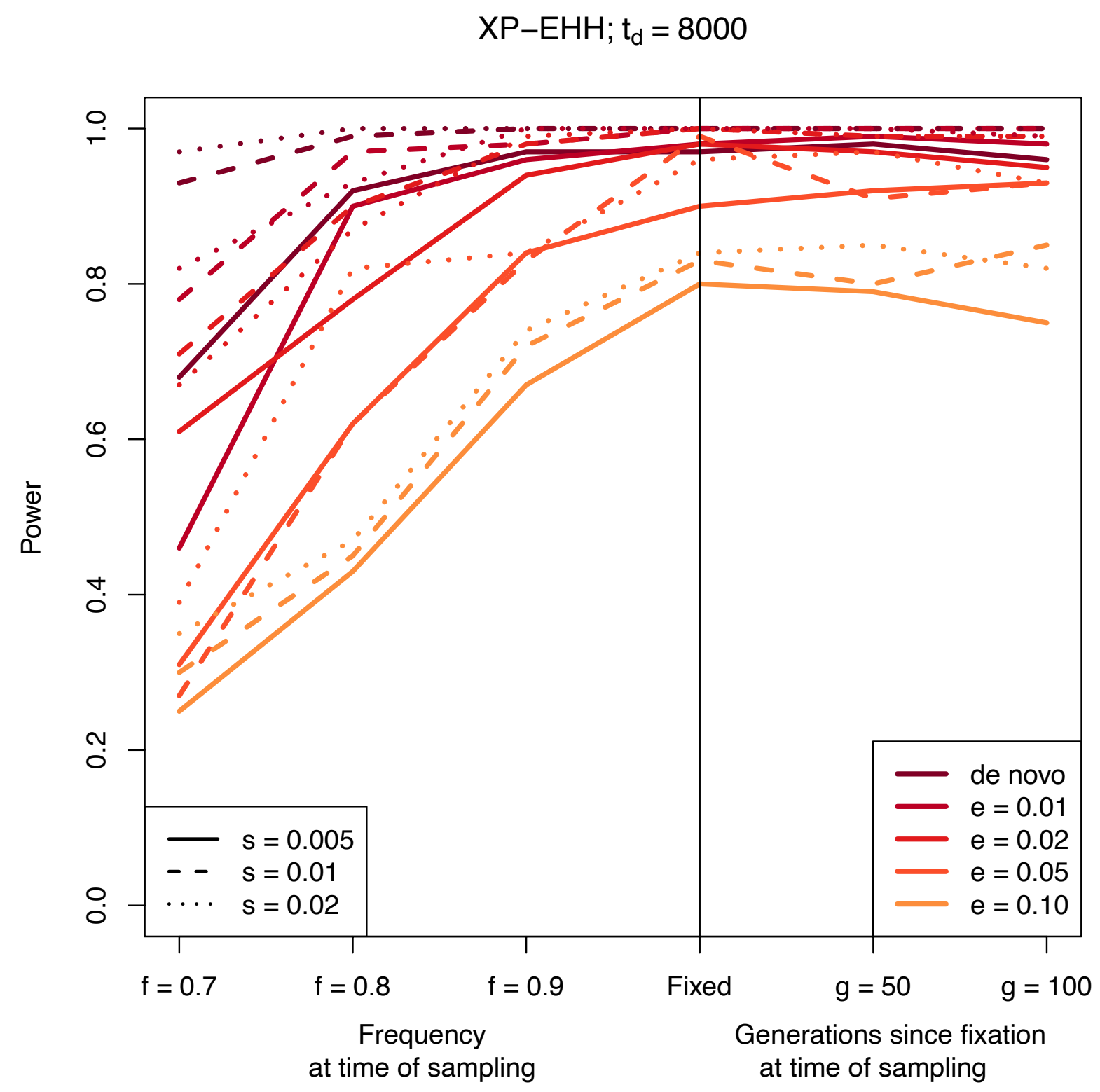

Figure S26. Demo 4 XP-EHH $t_{d}=8000$ power curves. $s$ is the selection coefficient, $f$ is the

323 frequency of the adaptive allele at time of sampling, $g$ is the number of generations at time of

324 sampling since fixation, $e$ is the frequency at which selection began, and $t_{d}$ is the time in

325 generations since the two populations diverged. 


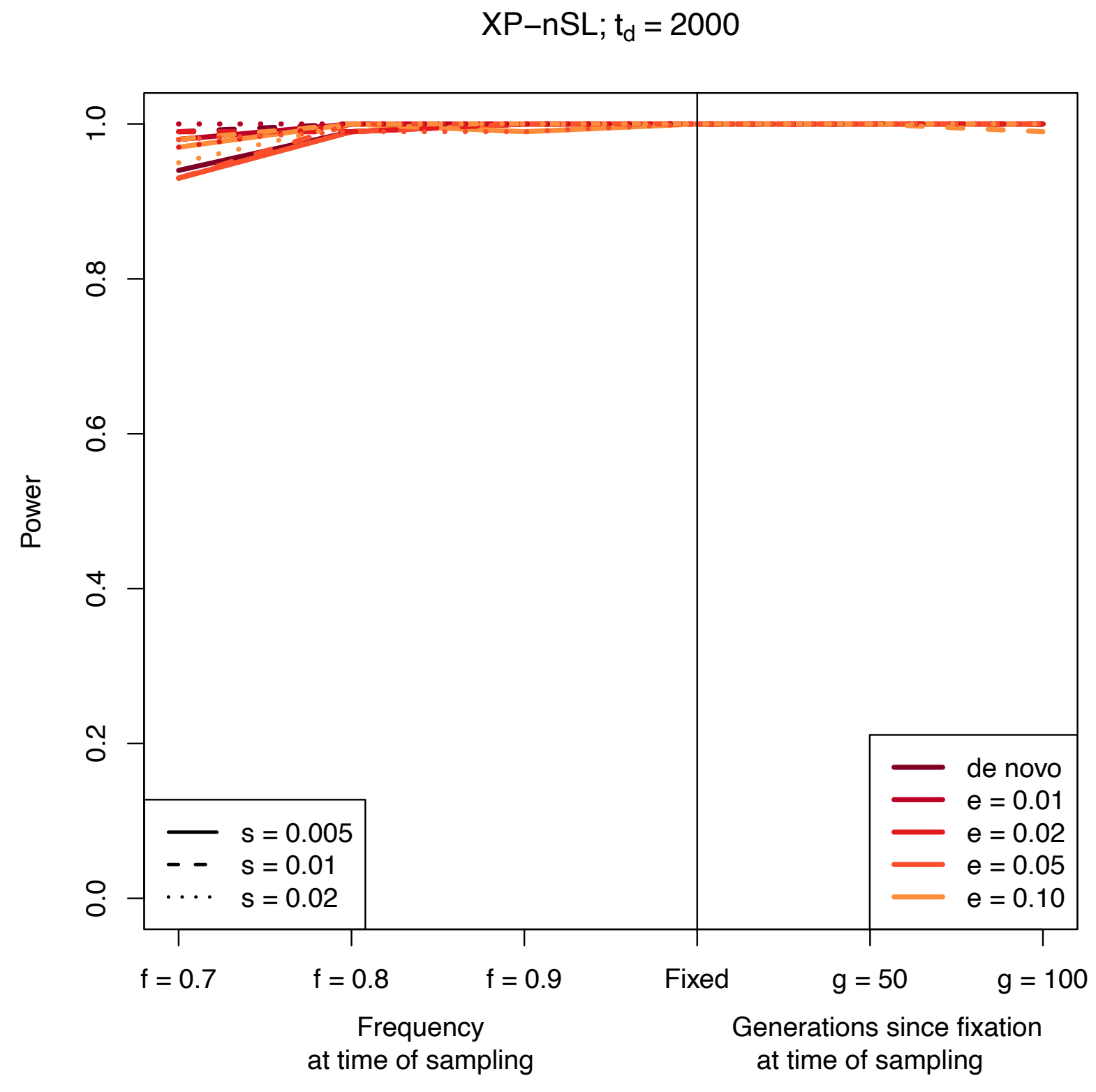

327 Figure S27. Demo 4 XP-nSL $t_{d}=2000$ power curves. $s$ is the selection coefficient, $f$ is the

328 frequency of the adaptive allele at time of sampling, $g$ is the number of generations at time of

329 sampling since fixation, $e$ is the frequency at which selection began, and $t_{d}$ is the time in

330 generations since the two populations diverged. 




Figure S28. Demo 4 XP-nSL $t_{d}=8000$ power curves. $s$ is the selection coefficient, $f$ is the

333 frequency of the adaptive allele at time of sampling, $g$ is the number of generations at time of

334 sampling since fixation, $e$ is the frequency at which selection began, and $t_{d}$ is the time in

335 generations since the two populations diverged. 


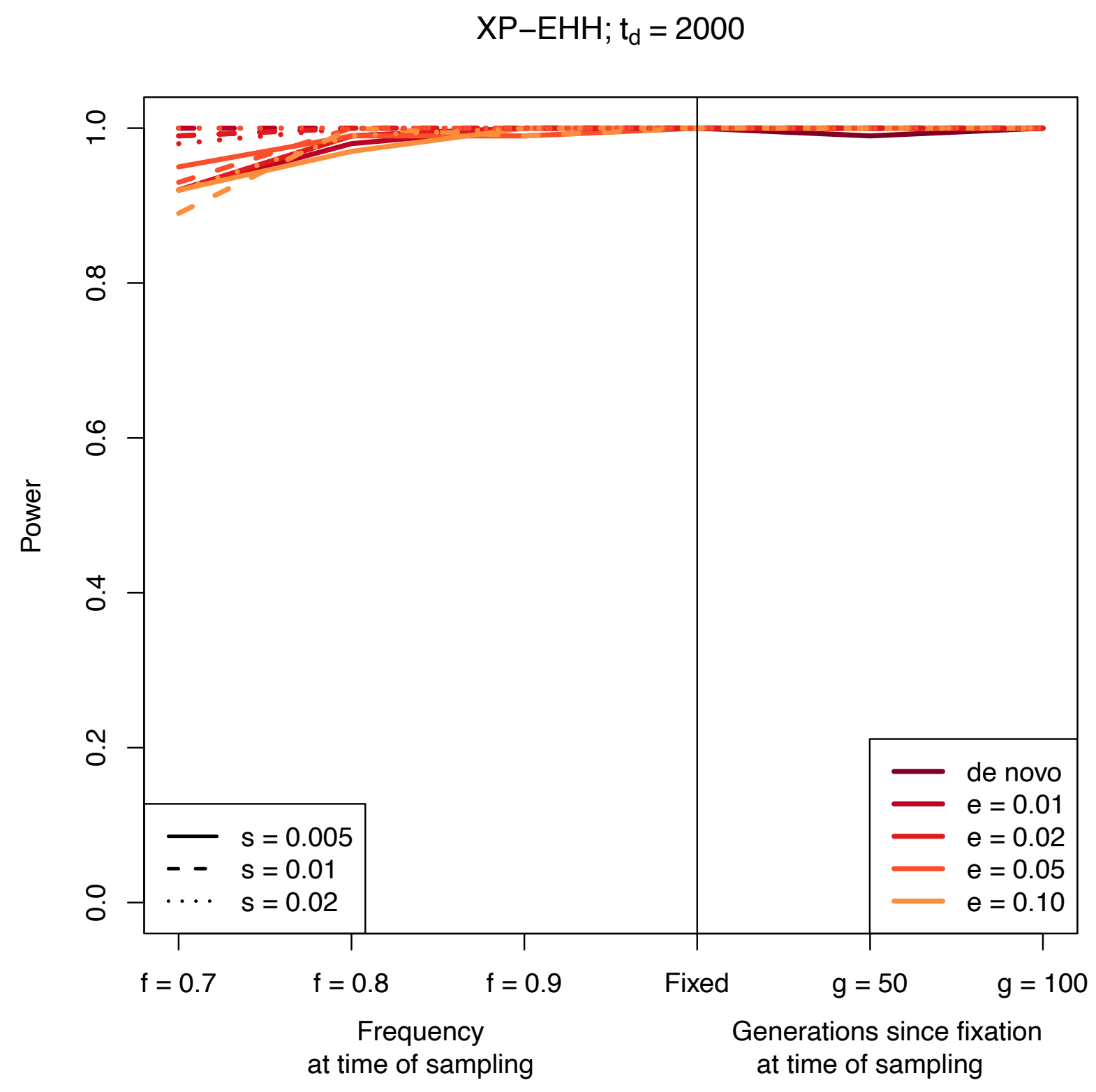

Figure S29. Demo $5 \mathrm{XP}-\mathrm{EHH} t_{d}=2000$ power curves. $s$ is the selection coefficient, $f$ is the

338 frequency of the adaptive allele at time of sampling, $g$ is the number of generations at time of

339 sampling since fixation, $e$ is the frequency at which selection began, and $t_{d}$ is the time in

340 generations since the two populations diverged. 


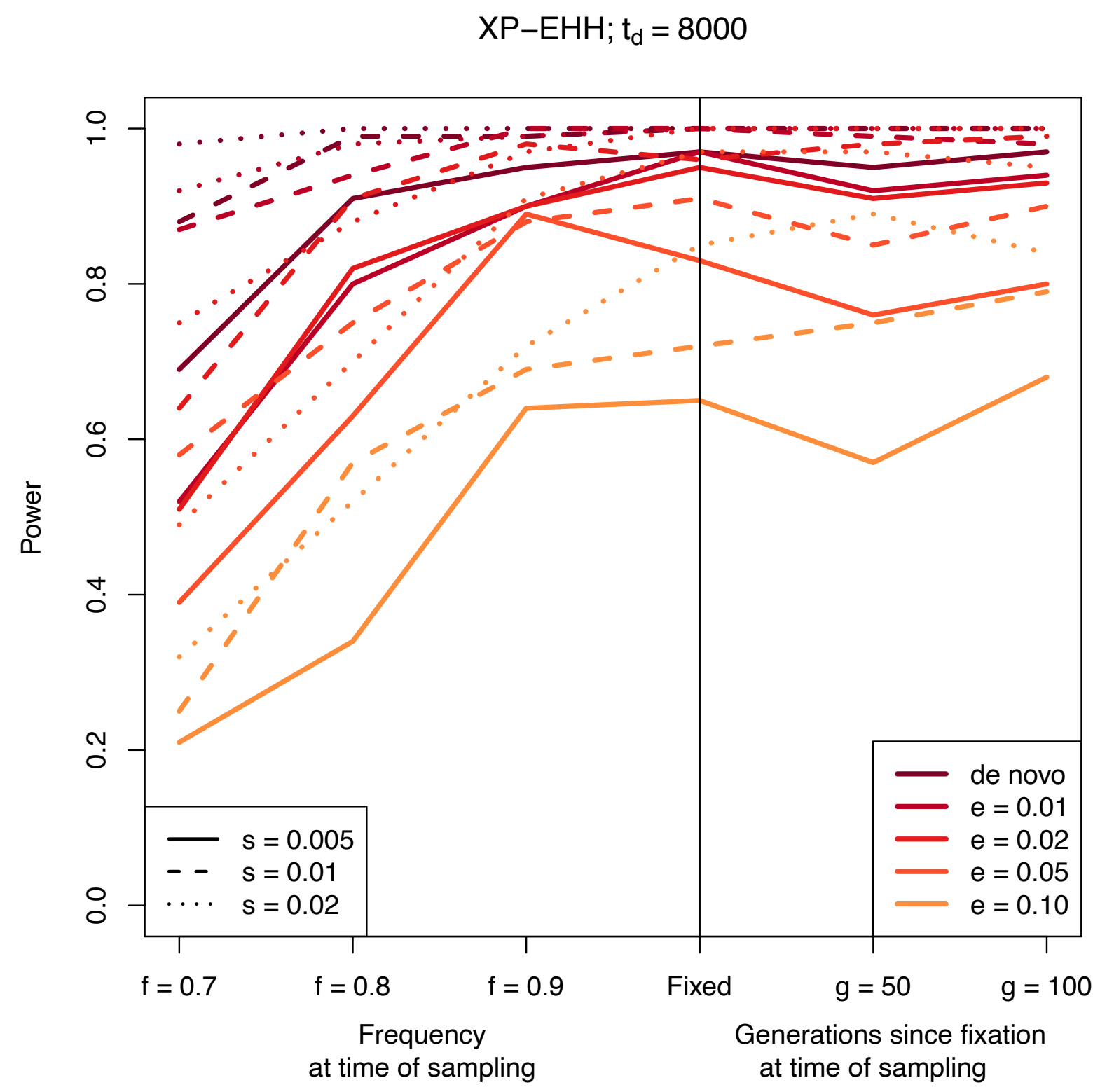

Figure S30. Demo $5 \mathrm{XP}-\mathrm{EHH} t_{d}=8000$ power curves. $s$ is the selection coefficient, $f$ is the

343 frequency of the adaptive allele at time of sampling, $g$ is the number of generations at time of

344 sampling since fixation, $e$ is the frequency at which selection began, and $t_{d}$ is the time in

345 generations since the two populations diverged. 


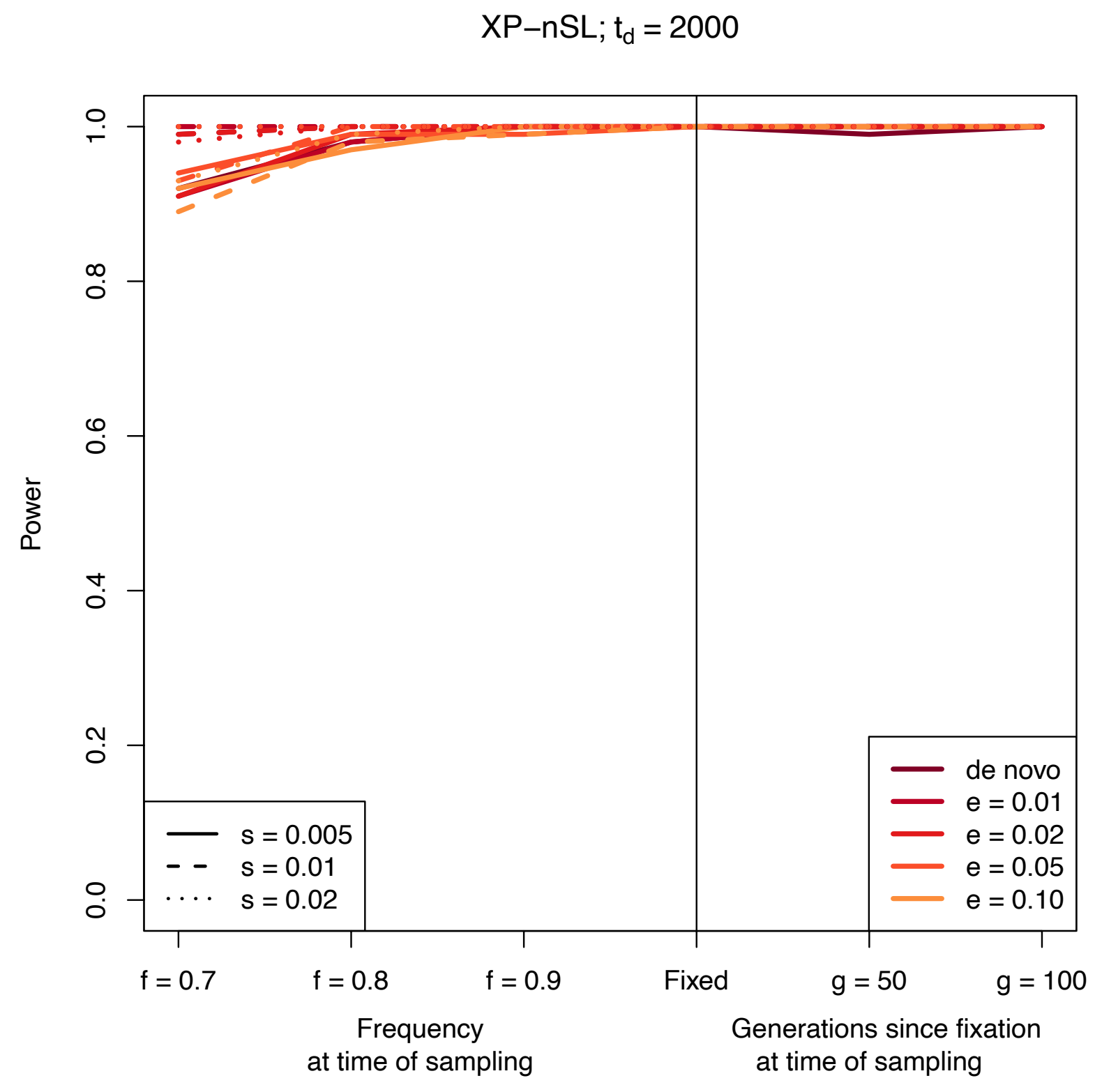

Figure S31. Demo 5 XP-nSL $t_{d}=2000$ power curves. $s$ is the selection coefficient, $f$ is the

348 frequency of the adaptive allele at time of sampling, $g$ is the number of generations at time of

349 sampling since fixation, $e$ is the frequency at which selection began, and $t_{d}$ is the time in

350 generations since the two populations diverged. 




Figure S32. Demo 5 XP-nSL $t_{d}=8000$ power curves. $s$ is the selection coefficient, $f$ is the

353 frequency of the adaptive allele at time of sampling, $g$ is the number of generations at time of

354 sampling since fixation, $e$ is the frequency at which selection began, and $t_{d}$ is the time in

355 generations since the two populations diverged. 
361 Table 1. Demographic history parameters for simulations.

\begin{tabular}{|l|c|c|c|c|c|c|}
\hline & $N_{A}$ & $N_{0}$ at split & $N_{0}$ at present & $N_{1}$ at split & $N_{1}$ at present & $t_{d}$ \\
\hline Demo 1 & 10,000 & 10,000 & 10,000 & 10,000 & 10,000 & $2,000 / 4,000 / 8,000$ \\
\hline Demo 2 & 10,000 & 10,000 & 10,000 & 5,000 & 5,000 & $2,000 / 4,000 / 8,000$ \\
\hline Demo 3 & 10,000 & 5,000 & 5,000 & 10,000 & 10,000 & $2,000 / 4,000 / 8,000$ \\
\hline Demo 4 & 10,000 & 10,000 & $50,000^{\dagger}$ & 10,000 & 10,000 & $2,000 / 4,000 / 8,000$ \\
\hline Demo 5 & 10,000 & 10,000 & 10,000 & 10,000 & $50,000^{\dagger}$ & $2,000 / 4,000 / 8,000$ \\
\hline
\end{tabular}

362

363

364

365

366

367

368

369

370

371

372

373

374

375

376

377

378

379

380

381

382

†The reached via exponential growth starting 2,000 generations ago.

Table S1. False positive rate computed from neutral simulations for varying $t_{d}$ and demographic history.

\begin{tabular}{|c|l|l|l|l|}
\hline \multirow{3}{*}{ iHS } & \multicolumn{1}{|c|}{$t_{d}=2000$} & \multicolumn{1}{c|}{$t_{d}=4000$} & $t_{d}=8000$ \\
\hline \multirow{3}{*}{$\mathrm{nSL}$} & Demo 1 & 0.013 & 0.1 & 0.009 \\
\cline { 2 - 5 } & Demo 3 & 0.007 & 0.013 & 0.007 \\
\cline { 2 - 5 } & Demo 4 & 0.015 & 0.018 & 0.008 \\
\hline \multirow{5}{*}{ XP-EHH } & Demo 1 & 0.01 & 0.015 & 0.008 \\
\cline { 2 - 5 } & Demo 3 & 0.008 & 0.011 & 0.007 \\
\cline { 2 - 5 } & Demo 4 & 0.014 & 0.021 & 0.014 \\
\cline { 2 - 5 } & Demo 1 & 0.013 & 0.013 & 0.016 \\
\cline { 2 - 5 } & Demo 2 & 0.017 & 0.009 & 0.015 \\
\cline { 2 - 5 } & Demo 3 & 0.01 & 0.011 & 0.012 \\
\cline { 2 - 5 } & Demo 4 & 0.012 & 0.014 & 0.014 \\
\hline \multirow{5}{*}{ XP-nSL } & Demo 5 & 0.011 & 0.012 & 0.013 \\
\cline { 2 - 5 } & Demo 1 & 0.014 & 0.011 & 0.013 \\
\cline { 2 - 5 } & Demo 2 & 0.019 & 0.011 & 0.012 \\
\cline { 2 - 5 } & Demo 4 & 0.011 & 0.011 & 0.014 \\
\cline { 2 - 5 } & Demo 5 & 0.012 & 0.012 & 0.014 \\
\hline
\end{tabular}

\section{References}

Colonna V, Ayub Q, Chen Y, Pagani L, Luisi P, Pybus M, Garrison E, Xue Y, Tyler-Smith C, Genomes Project C, et al. 2014. Human genomic regions with exceptionally high levels of population differentiation identified from 911 whole-genome sequences. Genome Biol 15:R88.

Crawford NG, Kelly DE, Hansen MEB, Beltrame MH, Fan S, Bowman SL, Jewett E, Ranciaro A, Thompson S, Lo Y, et al. 2017. Loci associated with skin pigmentation identified in African populations. Science 358.

DeGiorgio M, Szpiech ZA. 2021. A spatially aware likelihood test to detect sweeps from haplotype distributions. bioRxiv:2021.2005.2012.443825.

Ferrer-Admetlla A, Liang M, Korneliussen T, Nielsen R. 2014. On detecting incomplete soft or hard selective sweeps using haplotype structure. Mol Biol Evol 31:1275-1291.

Harris AM, DeGiorgio M. 2020. A likelihood approach for uncovering selective sweep signatures from haplotype data. Mol Biol Evol. 
Harris AM, Garud NR, DeGiorgio M. 2018. Detection and Classification of Hard and Soft Sweeps from Unphased Genotypes by Multilocus Genotype Identity. Genetics 210:14291452.

Kern AD, Schrider DR. 2016. Discoal: flexible coalescent simulations with selection. Bioinformatics 32:3839-3841.

Lu K, Wei L, Li X, Wang Y, Wu J, Liu M, Zhang C, Chen Z, Xiao Z, Jian H, et al. 2019. Wholegenome resequencing reveals Brassica napus origin and genetic loci involved in its improvement. Nat Commun 10:1154.

Meier JI, Marques DA, Wagner CE, Excoffier L, Seehausen 0. 2018. Genomics of Parallel Ecological Speciation in Lake Victoria Cichlids. Mol Biol Evol 35:1489-1506.

Nedelec Y, Sanz J, Baharian G, Szpiech ZA, Pacis A, Dumaine A, Grenier JC, Freiman A, Sams AJ, Hebert S, et al. 2016. Genetic Ancestry and Natural Selection Drive Population Differences in Immune Responses to Pathogens. Cell 167:657-669 e621.

Sabeti PC, Varilly P, Fry B, Lohmueller J, Hostetter E, Cotsapas C, Xie X, Byrne EH, McCarroll SA, Gaudet R, et al. 2007. Genome-wide detection and characterization of positive selection in human populations. Nature 449:913-918.

Salmon P, Jacobs A, Ahren D, Biard C, Dingemanse NJ, Dominoni DM, Helm B, Lundberg M, Senar JC, Sprau P, et al. 2021. Continent-wide genomic signatures of adaptation to urbanisation in a songbird across Europe. Nat Commun 12:2983.

Szpiech ZA, Hernandez RD. 2014. selscan: an efficient multithreaded program to perform EHH-based scans for positive selection. Mol Biol Evol 31:2824-2827.

Szpiech ZA, Novak TE, Bailey NP, Stevison LS. 2021. Application of a novel haplotype-based scan for local adaptation to study high-altitude adaptation in rhesus macaques. Evol Lett 5:408-421.

Voight BF, Kudaravalli S, Wen X, Pritchard JK. 2006. A map of recent positive selection in the human genome. Plos Biology 4:e72.

Zhang SJ, Wang GD, Ma P, Zhang LL, Yin TT, Liu YH, Otecko NO, Wang M, Ma YP, Wang L, et al. 2020. Genomic regions under selection in the feralization of the dingoes. Nat Commun 11:671.

Zoledziewska M, Sidore C, Chiang CWK, Sanna S, Mulas A, Steri M, Busonero F, Marcus JH, Marongiu M, Maschio A, et al. 2015. Height-reducing variants and selection for short stature in Sardinia. Nat Genet 47:1352-1356. 